hep-th/0005036

MIT-CTP-2975

MRI-PHY/P20000430

\title{
D-branes as Tachyon Lumps in String Field Theory
}

\author{
Nicolas Moeller ${ }^{a}$, Ashoke Sen $^{b}$ and Barton Zwiebach ${ }^{c}$ \\ ${ }^{a, c}$ Center for Theoretical Physics \\ Massachusetts Institute of Technology \\ Cambridge, MA 02139, USA \\ E-mail: moeller@pierre.mit.edu,zwiebach@mitlns.mit.edu \\ ${ }^{b}$ Mehta Research Institute of Mathematics \\ and Mathematical Physics, Chhatnag Road, \\ Jhoosi, Allahabad 211019, INDIA \\ E-mail: asen@thwgs.cern.ch, sen@mri.ernet.in
}

\begin{abstract}
It has been conjectured that the tachyonic lump solution of the open bosonic string field theory describing a D-brane represents a D-brane of one lower dimension. We place the lump on a circle of finite radius and develop a variant of the level expansion scheme that allows systematic account of all higher derivative terms in the string field theory action, and gives a calculational scheme that can be carried to arbitrary accuracy. Using this approach we obtain lump masses that agree with expected D-brane masses to an accuracy of about $1 \%$. We find convincing evidence that in string field theory the lump representing a D-brane is an extended object with a definite profile. A gaussian fit to the lump gives a 6 -sigma size of $9.3 \sqrt{\alpha^{\prime}}$. The level truncation scheme developed here naturally gives rise to an infrared and ultraviolet cut-off, and may be useful in the study of quantum string field theory.
\end{abstract}




\section{Table of Contents}

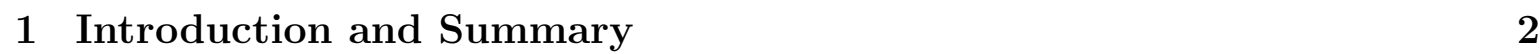

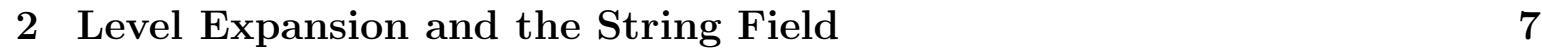

2.1 Modified level expansion . . . . . . . . . . . . . . . . . . . . . . . . 7

2.2 Background Independent String Field . . . . . . . . . . . . . . . . . . . 9

2.3 Mass of the lump . . . . . . . . . . . . . . . . . . . . . . 13

2.4 Setup and Sample Computations . . . . . . . . . . . . . . . 15

3 Calculating the action in the Level Expansion for $R=\sqrt{3} \quad 18$

3.1 The terms in the potential . . . . . . . . . . . . . . . . . . . . . . . 20

3.2 Potentials at various truncation levels and mass calculations . . . . . . 23

4 Tachyon Lump at Other Radii 28

$4.1 \quad R>\sqrt{3} \ldots \ldots \ldots \ldots \ldots$. . . . . . . . . . . . . . . . . 28

$4.2 R<\sqrt{3} \ldots \ldots \ldots \ldots \ldots \ldots$

4.3 Size of the lump . . . . . . . . . . . . . . . . . . . . . . . 33

5 Conclusions and Open Questions 35

\section{Introduction and Summary}

The 26-dimensional critical bosonic string theory admits Dirichlet $p$-branes (D- $p$-branes) for all $p \leq 26$. Each of these D-p-branes admits a tachyonic mode $T$ of mass ${ }^{2}=-1$, in units where the tension of the fundamental string is equal to $(2 \pi)^{-1}\left(\alpha^{\prime}=1\right)$. It has been conjectured that the potential for the tachyon field has a non-trivial translationally invariant (local) minimum at some value $T_{v a c}$ where the sum of the tachyon potential and the tension of the original brane vanishes [1]. Thus at $T=T_{\text {vac }}$ the total energy density vanishes, and hence this configuration can be identified as the vacuum of the closed string theory without any D-branes. It has also been conjectured that although this vacuum does not have any perturbative open string excitations, it contains lump-like soliton configurations which approach the vacuum $T=T_{\text {vac }}$ asymptotically far away from the core of the soliton and represent D-branes of lower dimension [2, 1]. 
Similar conjectures have also been made involving the tachyon living on the coincident D-brane anti-D-brane pair, or on a non-BPS D-brane of type IIA and IIB superstring theories [3, 4, 5, 6, 0, 8].

Various pieces of evidence for these conjectures have been found in both the first [2, 1, 3, 4, 5], and second [9, 10, 11, 12, 13, 14, 15, 16, 17] quantized string theory, and also using AdS/CFT correspondence [18, 19]. The first quantized description has been successful in verifying the conjectures relating the tachyonic solitons to lower dimensional D-branes, but it can only supply indirect evidence for the equivalence between the (local) minimum of the tachyon potential and the vacuum without a D-brane. On the other hand, the second quantized description - open string field theory [20] can provide direct evidence for this conjecture by explicitly computing the (negative) value of the tachyon potential at the minimum and comparing it with the tension of the original D-brane system. Although open string field theory contains infinite number of fields, and the problem of finding a translationally invariant stationary point of the potential involves solving the equations of motion of the infinite number of zero momentum modes of these fields, the calculations are made feasible by using the level expansion scheme proposed by Kostelecky and Samuel [21]. The procedure is as follows. Using the correspondence between the modes of the string field and states in the conformal field theory describing the first quantized string, we define the level of a mode of the string field as the difference between the $\widehat{N}$ eigenvalue of the first quantized string state representing this mode, and the $\widehat{N}$ eigenvalue of the state representing the zero momentum tachyon, where $\widehat{N}$ is the total 'number operator' of the matter and ghost system. The level truncation scheme to order $(M, N)$ then corresponds to an approximation in which we keep in the string field theory action all modes of level $\leq M$, and all interaction terms for which the sum of the levels of all the modes appearing in the term is $\leq N$. This gives a potential (which, for a static field configuration, is just the negative of the action up to a normalization constant) with finite number of fields and a finite number of terms. Thus we can find its extremum and calculate its value at the extremum. The larger the values of $(M, N)$, the larger is the number of modes and the number of terms in the potential, and the better is the accuracy.

The calculation of ref. [21] for the tachyon potential was revisited and extended in [9] in terms of background independent fields. It was shown there that the total negative 
potential energy at the stationary point cancels the energy of the D-brane represented by the string field theory to an accuracy of $<1.5 \%$ at the level $(4,8)$ approximation. This calculation was extended in ref. [11] to level $(10,20)$. At this level the contribution from the tachyon potential was found to cancel the tension of the D-brane to an accuracy of about .1\%. Similar calculations have also been performed [14, 15, 16, 17] in open superstring field theory [22, 23, 24]. At the level $(2,4)$ approximation the tachyon potential has been shown to cancel about $90 \%$ of the tension of the original brane configuration.'

The success of string field theory in verifying the conjecture relating the translationally invariant stationary point of the tachyon potential and the vacuum without any D-brane encourages one to ask whether string field theory can also be used in studying the conjectured relation between the tachyonic lump solutions and lower dimensional D-branes. This study was initiated by Harvey and Kraus [12]. In this paper they started with the level $(0,0)$ contribution to the tachyon potential in open bosonic string field theory on a D-p-brane, and identified a 'bounce solution' in this field theory as the $\mathrm{D}-(p-1)$ brane. At this level the tension associated with this solution turns out to be about $78 \%$ of the known value of the $\mathrm{D}$ - $(p-1)$-brane tension. This result receives correction not only from the higher level fields, but also from the momentum dependence of the interaction terms which were neglected in the initial analysis. While there is no systematic expansion scheme for taking into account these momentum dependent corrections, a naive expansion of the interaction term in powers of momentum, keeping only the zeroth and first order terms, reduced the tension of the soliton to about $70 \%$ of the conjectured answer. On the other hand, taking into account the correction to the potential to level $(2,4)$ increased the answer back to about $82 \%$ of the conjectured answer. A systematic method for taking into account the momentum dependent terms in the interaction was suggested in ref. [13], but this procedure did not give rise to an appreciable change in the tension of the lump. A similar analysis has also been carried out for solitons in the open superstring field theory [15, 25, 26]. Although the answer turns out to be close to the expected answer, it is likely to be an accidental result, as there is no reason to assume that the corrections due to the momentum dependent terms are small in this case.

\footnotetext{
${ }^{1}$ At present there seems to be some disagreement between refs. [16] and [17] about the level $(2,4)$ results.
} 
The purpose of this paper will be to develop a systematic approximation scheme for studying these solitons in string field theory and calculating their tension. We shall focus on the codimension one lump on a $\mathrm{D} p$-brane of the bosonic string theory - which is conjectured to be equivalent to a D- $(p-1)$-brane - but it will become clear that the scheme is general enough to be applicable to the study of higher codimension solitons, as well as to solitons in superstring field theory. In the case of a codimension one soliton, we are dealing with a field configuration on the $\mathrm{D} p$ brane which depends on only one of the spatial coordinates (say $x$ ) on the brane, and is independent of time, as well as the other $(p-1)$ spatial coordinates. We study this problem by compactifying the coordinate $x$ on a circle of radius $R$ instead of letting it span the whole real line. In this case, since all field configurations must be periodic in $x$, we can decompose all fields into modes carrying discrete momenta along $x$ in units of $(1 / R)$, and the solitonic field configuration that we are looking for must be obtained as an appropriate superposition of these modes. We can now define the level of any such mode as the difference between the $L_{0}$ eigenvalue of the first quantized string state representing this mode, and that of the zero momentum tachyon state, where $L_{0}$ denotes the zeroth component of the Virasoro generator of the combined matter ghost system. This allows us to define a level $(M, N)$ approximation to the potential exactly as before. Working with the potential up to a given level, we can now look for $x$ dependent solutions of the string field equations by extremizing the potential with respect to the modes appearing in the potential to this level.

This is precisely the procedure we follow in this paper for studying the tachyonic lump solution on a D-p-brane. We study this problem for various radii at various levels of approximation, and compare the tension of the lump with the tension of a D- $(p-1)$-brane. The results for the tension of the lump turn out to be remarkably close to the known tension of the $\mathrm{D}$ - $(p-1)$-brane. Whereas for $R=\sqrt{3}$ and $\sqrt{15 / 2}$ we are able to get a lump tension within $1 \%$ of the tension of the D-( $p-1)$-brane, for larger $\operatorname{radii}(R=\sqrt{12}$ and $\sqrt{35 / 2})$ we get answers within $3 \%$ of the expected answer. We also compare the profile of the tachyon field corresponding to the lump for different values

\footnotetext{
${ }^{2}$ Since for the zero momentum states the eigenvalue of the number operator is the same as the $L_{0}$ eigenvalue of the state, the two prescriptions agree for these states.

${ }^{3}$ The discretization of the momentum is reminiscent of the procedure followed in ref. [13], although the precise relationship between these two approaches is not clear.
} 
of $R$, - obtained by superposition of $\cos (n x / R)$ for integer $n$ - and find remarkable agreement between the profiles for different values of $R$.

At this point we should note that the problem of formation of the tachyonic lump on a circle was addressed using the first quantized approach in ref. [27]. There a renormalization group analysis was used to show that the mass of the tachyonic lump on a $\mathrm{D}$ - $p$-brane is indeed equal to that of a $\mathrm{D}$ - $(p-1)$-brane. In view of this result one might ask whether the string field theory analysis carried out in this paper gives any new insight into this problem. To this end, we note, first of all, that the relationship between the renormalization group analysis in the first quantized approach, and the string field theory analysis based on the level truncation scheme, is as yet quite unclear, and hence it is certainly illuminating to independently verify the equivalence of the $\mathrm{D}-(p-1)$ brane, and the tachyonic lump on the D-p-brane in string field theory. Furthermore, string field theory provides us with a definite picture of the tachyon profile as superposition of $\cos (n x / R)$ for different $n$ with definite coefficients. In contrast the analysis based on the renormalization group flow only tells us that a perturbation by the leading relevant operator $\cos (x / R)$ takes the original $\mathrm{D}$-p-brane to a $\mathrm{D}$ - $(p-1)$-brane, and does not tell us how the higher harmonics mix with $\cos (x / R)$ to produce the soliton. Indeed most of the higher harmonics correspond to irrelevant perturbation, and hence their coefficients vanish in the infra-red.] Furthermore, the rigorous results of ref. [28] have not yet been generalized to superstring theory. Thus we believe that despite the exact results based on the renormalization group analysis of the first quantized theory, the present analysis throws new light on the tachyonic soliton solutions.

The rest of the paper is organized as follows. In section 2 we outline the general procedure of level expansion scheme of the string field theory, discuss the possibility of restricting the string field to a background independent subspace for studying the lump solution, and give details of computation of a few terms in the potential. In section 3 we give in detail the results for the potential, the lump solution and its energy for a specific radius $R=\sqrt{3}$. We also compare the profile of the lump at different levels of approximation. In section t we give the results for several other radii, both larger

\footnotetext{
${ }^{4}$ This followed earlier work of ref. [28] on the renormalization group flow of the two dimensional field theory under a perturbation corresponding to switching on a tachyon background proportional to $\cos (x / R)$.

${ }^{5}$ Presumably if we could determine the exact location of the infrared fixed point in the space of coupling constants, then the shape of the lump will be determined in this approach.
} 
$(\sqrt{15 / 2}, \sqrt{12}$ and $\sqrt{35 / 2})$ and smaller $(\sqrt{11 / 10})$ than $\sqrt{3}$, and compare the profile of the lump for each radii with the profile at $R=\sqrt{3}$. We conclude in section 0 by discussing possible generalization of this analysis and some speculations.

\section{Level Expansion and the String Field}

In this section we will set up a variant of the level expansion method to deal with the problem of finding the profile and mass of the tachyon lump in string field theory. As reviewed in the introduction, such method is desirable as previous computations of lump masses in string field theory have not been very accurate. After explaining this method we will discuss the background independent expansion of the string field suitable for the problem. Then we discuss two methods for estimating the lump mass. We conclude by showing a few samples of typical calculations needed to evaluate the string field action for the lump.

\subsection{Modified level expansion}

When calculating the tachyon potential in search for a spacetime independent vacuum state, all spacetime fields are set to constants, and the evaluation of the string field action does not require the inclusion of terms with spacetime derivatives. The string field is at zero momentum and is thus built by a superposition of zero momentum states times constants representing the zero momentum modes of the spacetime fields. The states are built by acting on a zero-momentum vacuum with oscillators of the relevant conformal field theory (CFT). In this case the level expansion was defined as follows [21. Let $\widehat{N}$ be the number operator, representing the contribution to $L_{0}$ from the system of matter and ghost oscillators. Let $N_{0}(=-1)$ denote the eigenvalue of $\widehat{N}$ for the zero momentum tachyon: $\widehat{N}\left|T_{0}\right\rangle=N_{0}\left|T_{0}\right\rangle$. For a given state $\left|\Phi_{i}\right\rangle$, with number eigenvalue $N_{i}\left(\widehat{N}\left|\Phi_{i}\right\rangle=N_{i}\left|\Phi_{i}\right\rangle\right)$ we define the level $l\left(\Phi_{i}\right)$ of the state $\left|\Phi_{i}\right\rangle$ as

$$
l\left(\Phi_{i}\right) \equiv N_{i}-N_{0} .
$$

As defined, level is a dimensionless number. For the case of bosonic string theory the levels are all integers while for NS superstrings they can also be half integral. We now define the level $(M, N)$ approximation to the action as follows: 
- We keep only those fields with level $\leq M$.

- We keep only those terms in the action for which the sum of the levels of all the fields in the term is $\leq N$.

In order that the quadratic term of all fields with level $\leq M$ are kept in the action, we must have $N \geq 2 M$. While variants are possible, it seems most effective when calculating any physical object to use its level $(M, 2 M)$ approximation, as experience shows that increasing the number of terms in the potential keeping the number of fields fixed does not improve the results very much. While there is yet no theoretical explanation for the convergence of the level expansion, the numerical evidence collected thus far is impressive.

Consider now the problem at hand. While all of our discussion applies to soliton solutions on non-BPS D-branes, and D-brane anti- D-brane pairs of superstring theory, we will consider here explicitly only the case of the unstable D-branes of bosonic string theory. Consider therefore, an unstable bosonic D-brane extending over a number of spatial dimensions. We now wish to select one of these dimensions, call it $x$ and construct a tachyon lump such that the solution depends only on the $x$-coordinate. (Again our discussion applies to lumps depending on more than one coordinates, but we shall not analyze these cases here.) As the lump is not invariant under translation along $x$, we now need to include $x$-momentum modes in the string field expansion and $x$-derivatives, or $x$-momentum dependent terms in the string field action. In order to do this systematically we compactify $x$ over a circle of radius $R$, namely $x \sim x+2 \pi R$. This quantizes the $x$-momentum as $p_{x}=n / R$ for integer $n$. For each of the zero momentum states $\left|\Phi_{i}\right\rangle$ we had before, we now have discrete states of the type $\left|\Phi_{i, n}\right\rangle$ that only differ by the fact that they are built on vacua having $x$-momentum $n / R$. For such states there is a natural generalization of the level. This is the difference between the $L_{0}$ eigenvalue of the state and that of the zero momentum tachyon, where $\left\{L_{n}\right\}$ denote the Virasoro generators of the combined matter and ghost system. This is because (with $\alpha^{\prime}=1$ ) we have that $L_{0}=p_{x}^{2}+\widehat{N}$. For zero momentum this is just the previous definition. Still denoting by $N_{i}$ the number eigenvalue of $\left|\Phi_{i, n}\right\rangle$ we have

$$
l\left(\Phi_{i, n}\right)=L_{0}\left(\Phi_{i, n}\right)-L_{0}\left(T_{0}\right)=\frac{n^{2}}{R^{2}}+N_{i}-N_{0} .
$$


The level is still a dimensionless number as $R$ here is measured in units of $\sqrt{\alpha^{\prime}}$ (which has been set to one). We can now define the level $(M, N)$ approximation for the action exactly as before. Since the $L_{0}$ eigenvalue of a state plays a crucial role in the conformal map that inserts the state into the disk representing the interaction terms in the action, this is a natural generalization of the level truncation scheme of ref. [21]. This paper will present evidence that this modified version of the level truncation scheme also works very well.

In calculating in this setup in the level $(M, 2 M)$ approximation for any given radius we will have to include states $\left|\Phi_{i}\right\rangle \equiv\left|\Phi_{i, 0}\right\rangle$ and "harmonics" $\left|\Phi_{i, n}\right\rangle$, and clearly the condition $l\left(\Phi_{i, n}\right) \leq M$ will give an upper bound on $n$ for each $i$. This also requires $l\left(\Phi_{i, 0}\right) \leq M$, and thus we have a finite number of modes to be included at a given level of approximation. Each term in the action including modes whose sum of levels does not exceed $2 M$ is computed exactly. It is manifest that in a cubic string field theory the level $(M, 2 M)$ approximation will only require a finite number of computations 0 .

\subsection{Background Independent String Field}

The general setup required to study a lump is similar to that developed in 29 to study the mass of the D-brane. To begin with, we assume that the background space-time is the product of a $(2+1)$ dimensional flat space-time, labelled by a pair of space-like coordinates $(x, y)$ and a time like coordinate $x^{0}$, and an arbitrary Euclidean manifold $\mathcal{M}$ described by a conformal field theory of central charge 23. We take the spatial direction $y$ to be non-compact, but $x$ to be compact with radius $R$. We let $X, Y$ and $X^{0}$ denote the three scalar fields on the string world-sheet associated with the coordinates $x, y$ and $x^{0}$.

We now consider a D-brane with the following properties. For an open string ending on the D-brane we put Neumann boundary condition on the fields $X$ and $X^{0}$ and Dirichlet boundary condition on the field $Y$. We leave the boundary condition on the fields associated with the coordinates on $\mathcal{M}$ arbitrary, with the only restriction that all the fields on which we put Neumann boundary condition are associated with compact

\footnotetext{
${ }^{6}$ This will also be the case for the NS superstring field theory discussed in ref. 14, 15, 16, 17]

${ }^{7}$ As in ref. [29], the extra non-compact direction $y$ with Dirichlet boundary condition provides a direction along which the brane can move, and we can calculate the tension of the brane by studying its motion in this direction.
} 
coordinates. This means that all directions tangential to the D-brane are compact, and hence the D-brane has finite mass. From the point of view of the full space-time, this D-brane describes a D- $p$ brane for some $p \geq 1$, with $(p-1)$ directions wrapped on an internal $(p-1)$ cycle of $\mathcal{M}$, and one direction wrapped on the circle of radius $R$ labelled by $x$. On the other hand from the point of view of an observer who only sees the $(2+1)$ dimensional space-time labelled by $\left(x, y, x^{0}\right)$, this system corresponds to a D1-brane wrapped on a circle of radius $R$. From now on we shall refer to this system as the D1-brane or the D-string; with its tension defined as the total energy per unit length along $x$. Of course, an ordinary D-string will be a special case of this system, obtained by putting Dirichlet boundary condition on all the fields associated with the coordinates on $\mathcal{M}$.

The dynamics of an open string with ends on this D-brane is described by a boundary conformal field theory of central charge 26, which is a direct sum of the boundary conformal field theories associated with the fields $X, Y, X^{0}$ and the manifold $\mathcal{M}$. We shall denote by $\operatorname{CFT}(X), \operatorname{CFT}(Y)$ and $\operatorname{CFT}\left(X^{0}\right)$ the boundary conformal field theories (each with central charge 1) associated with the fields $X, Y$ and $X^{0}$ respectively, and by $\operatorname{CFT}(\mathcal{M})$ the boundary conformal field theory with central charge 23 associated with the manifold $\mathcal{M}$. We also define

$$
\mathrm{CFT}^{\prime}=\mathrm{CFT}(Y) \oplus \mathrm{CFT}\left(X^{0}\right) \oplus \operatorname{CFT}(\mathcal{M})
$$

so that $\mathrm{CFT}^{\prime}$ has central charge 25. We denote by $L_{n}^{X}$ and $L_{n}^{\prime}$ the Virasoro generators of $\mathrm{CFT}(X)$ and $\mathrm{CFT}^{\prime}$ respectively. If we denote by $L_{n}^{\text {ghost }}$ the Virasoro generators of the ghost system, then the total Virasoro generators of the system will be given by $L_{n}=L_{n}^{\text {ghost }}+L_{n}^{X}+L_{n}^{\prime}$.

The compact direction $x$ corresponds to the direction in which we shall eventually form the lump. If we follow the normalization convention of ref. [9], then the tension $\mathcal{T}_{1}$ of the D-string described above is related to the coupling constant $g_{o}$ of the open string field theory describing the wrapped D-string by the relation:

$$
2 \pi R \mathcal{T}_{1}=\frac{1}{2 \pi^{2} g_{o}^{2}}
$$

In this normalization convention, a time independent string field configuration represented by a state $|\Phi\rangle=\Phi(0)|0\rangle$ in the Hilbert space of first quantized string theory, 
will have a potential

$$
\text { Potential }=-S(\Phi)=\frac{1}{g_{o}^{2}} \mathcal{V}(\Phi)=2 \pi R \mathcal{T}_{1} \cdot 2 \pi^{2} \mathcal{V}(\Phi)
$$

where

$$
\mathcal{V}(\Phi)=\frac{1}{2}\langle\Phi, Q \Phi\rangle+\frac{1}{3}\langle\Phi, \Phi * \Phi\rangle .
$$

Here $Q$ denotes the BRST charge, $\langle$,$\rangle denotes BPZ inner product between two states,$ and $*$ denotes the $*$-product of Witten's open bosonic string field theory [20].

A basis of states in $\mathrm{CFT}(\mathrm{X})$ is obtained by acting on $e^{i n X / R}(0)|0\rangle$ with the oscillators $\alpha_{-m}^{X}$ of $X$. It follows by a simple counting argument that an alternate basis can be formed out of the Verma module, containing states obtained by acting on $e^{i n X / R}(0)|0\rangle$ with the operators $L_{-m}^{X}$, as long as these states are all linearly independent. This is the case if there are no null states in the spectrum. The condition for the appearance of a null state is given by 30 ,

$$
\frac{n^{2}}{R^{2}}=\frac{(p-q)^{2}}{4} \rightarrow \frac{n}{R}=\frac{(p-q)}{2},
$$

where $p$ and $q$ are integers. Since $n$ is an integer, we can avoid null states for $n \neq 0$ with an appropriate choice of $R$. Even if we work with a value of $R$ for which there are null states, the choice of basis described above is good below the level where the first null state appears. From now on we shall restrict our analysis to situations where this choice of basis based on Verma module is good. In fact our explicit work in later sections will be based on $R$ values that are not rational, and thus there will be no null states for $n \neq 0$.

For $n=0$, however, there are null states and hence the basis of states obtained by applying $L_{-m}^{X}$ on $|0\rangle$ is not complete. For example, $L_{-1}^{X}|0\rangle$ is null, and this requires us to explicitly include the primary state $\alpha_{-1}^{X}|0\rangle$ in the basis. There are further null states in the Verma module over $\alpha_{-1}^{X}|0\rangle$, and hence there are new primary states at higher level which must be explicitly included in the basis. Let us denote by $\left\{\left|\varphi_{e}^{i}\right\rangle=\varphi_{e}^{i}(0)|0\rangle_{X}\right\}$ and $\left\{\left|\varphi_{o}^{i}\right\rangle=\varphi_{o}^{i}(0)|0\rangle_{X}\right\}$ the set of zero momentum primary states which are respectively even and odd under the reflection $X \rightarrow-X$. The complete basis of zero momentum states in $\mathrm{CFT}(\mathrm{X})$ is obtained by acting on $\left\{\left|\varphi_{e}^{i}\right\rangle\right\}$ and $\left\{\left|\varphi_{o}^{i}\right\rangle\right\}$ with $L_{-n}^{X}$ 's, and removing the null states. 
A generic string field configuration is represented by an arbitrary state in the Hilbert space $\mathcal{H}$ of ghost number one in the combined matter, ghost conformal field theory. We now claim that in order to discuss a lump along the $x$ coordinate, we can restrict the string field $|\Phi\rangle$ to a subspace $\widehat{\mathcal{H}}$ of $\mathcal{H}$, built by acting with the oscillators

$$
\left\{L_{-1}^{X}, L_{-2}^{X}, \cdots ; L_{-2}^{\prime}, L_{-3}^{\prime}, \cdots ; c_{1}, c_{-1}, c_{-2}, \cdots ; b_{-2}, b_{-3}, \cdots\right\}
$$

on the following primary states:

- The zero momentum even primaries $\varphi_{e}^{i}(0)|0\rangle$ (and removing the null states), and,

- The Fock vacuum states of the form

$$
\cos \left(\frac{n}{R} X(0)\right)|0\rangle=\frac{1}{2}\left(e^{i n X(0) / R}+e^{-i n X(0) / R}\right)|0\rangle=\frac{1}{2}\left(\left|\frac{n}{R}\right\rangle+\left|-\frac{n}{R}\right\rangle\right) \quad n \neq 0,
$$

where $|0\rangle$ is the $\mathrm{SL}(2, \mathrm{R})$ vacuum of the combined matter, ghost conformal field theory. A few points should be made. The Virasoro operator $L_{-1}^{\prime}$ is not required for it kills the above primary states (this is not the case for $L_{-1}^{X}$ ). $b_{-1}$ and $b_{0}$ also annihilate the vacuum $|0\rangle$, and hence have been omitted from the list. We have not included the oscillator $c_{0}$ because we work in the Siegel gauge, where all states must be annihilated by $b_{0}$. Finally we can restrict ourselves to states of even twist [31. This simply requires that the eigenvalue of the number operator $\widehat{N}$ must be odd (same as that for the tachyon).

In order to show that the above is a consistent truncation of the string field, one must show that there is no term in the action that couples a single state in $(\mathcal{H}-\widehat{\mathcal{H}})$ to a state in $\widehat{\mathcal{H}}$ via the quadratic term, or to a pair of states in $\widehat{\mathcal{H}}$ via the interaction term. This is readily done by listing the states in $(\mathcal{H}-\widehat{\mathcal{H}})$. We carry along all ghost oscillators and classify the states by their behavior under the matter operators. In this way we get the following disjoint sets:

- States with nonzero momentum $k_{0}$ along $X^{0}$.

- States obtained by acting with the oscillators in (2.8) on Fock vacua of the type $\sin \left(\frac{n X(0)}{R}\right)|0\rangle$, or on a state of the form $\varphi_{o}^{i}(0)|0\rangle$. 
- States obtained by acting with (2.8) on states that (i) have $k_{0}=0$, (ii) are nontrivial primaries of $\mathrm{CFT}^{\prime}$ (of dimension greater than zero, by unitarity), and (iii) are $\mathrm{CFT}(\mathrm{X})$ primaries.

It is manifest by momentum conservation that a state in the first set cannot couple to states in $\widehat{\mathcal{H}}$. The symmetry $X \rightarrow-X$ of $\mathrm{CFT}(\mathrm{X})$ insures that a state in the second set also cannot couple to states in $\widehat{\mathcal{H}}$. The same is true for the last set as Virasoro Ward identities can be used to show that a correlator involving two states in $\widehat{\mathcal{H}}$ and a state in the last set is proportional to the one point function of the $\mathrm{CFT}^{\prime}$ primary in question. Since this primary must have dimension greater than zero, its one point function vanishes. This completes our justification for the use of $\widehat{\mathcal{H}}$.

Since the choice of basis described above requires the use of the basis $\left\{\left|\varphi_{e}^{i}\right\rangle\right\}$, it will be useful to determine at which level the first zero momentum primary (other than the vacuum state) appears. For this we can compare the full partition function of CFT(X) for states even under $X \rightarrow-X$

$$
Z_{\text {even }}(q) \equiv \operatorname{Tr}_{\text {even }}\left(q^{L_{0}^{X}-\frac{1}{24}}\right)=\frac{1}{2} q^{-\frac{1}{24}}\left(\prod_{n=1}^{\infty} \frac{1}{1-q^{n}}+\prod_{n=1}^{\infty} \frac{1}{1+q^{n}}\right)
$$

with the Virasoro character for $(c=1, h=0)$ [30],

$$
\chi_{c=1, h=0}(q)=q^{-\frac{1}{24}} \prod_{n \geq 2} \frac{1}{1-q^{n}} .
$$

It can be easily checked that

$$
Z_{\text {even }}(q)-\chi_{c=1, h=0}(q)=q^{-\frac{1}{24}}\left(q^{4}+O\left(q^{5}\right)\right) .
$$

Thus the first non-trivial primary $\left|\varphi_{e}^{1}\right\rangle$ even under $X \rightarrow-X$ appears at level four. Indeed, the available descendents at this level, $L_{-4}^{X}|0\rangle, L_{-2}^{X} L_{-2}^{X}|0\rangle$, do not suffice to represent the nonvanishing $X$-even states $\alpha_{-3}^{X} \alpha_{-1}^{X}|0\rangle, \alpha_{-2}^{X} \alpha_{-2}^{X}|0\rangle,\left(\alpha_{-1}^{X}\right)^{4}|0\rangle$.

\subsection{Mass of the lump}

To begin with, the wrapped D- $p$ brane, which we have been calling a D-1 brane wrapped on a circle of radius $R$, has mass $2 \pi R \mathcal{T}_{1}$, where $\mathcal{T}_{1}$, as defined earlier, is the tension of this D1-brane. We want to compute the mass of the system in a situation where the 
tachyon field on this D1-brane develops a lump along a circle of radius $R$ (this direction is represented by the world sheet field $X$ ). If we denote by $\vec{T}$ the multicomponent string field configuration on the D1 brane, restricted to $\widehat{\mathcal{H}}$, then, using eq.(2.5), the rest mass energy plus potential energy of the D1 brane stretched on the circle can be written as

$$
E(D 1)=\mathcal{T}_{1}(2 \pi R)\left(1+2 \pi^{2} \mathcal{V}(\vec{T})\right)
$$

where $\mathcal{V}$ has been defined in eq.(2.6). Before condensation, $\vec{T}=0$ and $\mathcal{V}(\vec{T})=0$, and thus the energy formula correctly reproduces the mass of the D1-brane. Recall that for the nontrivial translationally invariant vacuum $\vec{T}_{v a c}$, one expects $\mathcal{V}\left(\vec{T}_{v a c}\right)=-1 /\left(2 \pi^{2}\right)$ and the energy formula correctly gives zero (as the D1 brane has disappeared). Using $\mathcal{V}\left(\vec{T}_{v a c}\right)=-1 /\left(2 \pi^{2}\right)$ we can write the energy formula as

$$
E(\mathrm{D} 1)=\mathcal{T}_{1} 2 \pi R \cdot 2 \pi^{2}\left(\mathcal{V}(\vec{T})-\mathcal{V}\left(\vec{T}_{\text {vac }}\right)\right)
$$

The mass of the tachyonic lump solution, represented by the configuration $\vec{T}_{\text {lump }}$, is obtained by replacing $\vec{T}$ by $\vec{T}_{\text {lump }}$ on the right hand sides of eqs. (2.13) or (2.14). This tachyonic lump on the D-string (wrapped D-p-brane) is conjectured to be equivalent to a D0-brane (a wrapped D- $(p-1)$-brane) of mass $\mathcal{T}_{0}$. With $\alpha^{\prime}=1$, the ratio of the tension of a D- $p$ brane and a D- $(p-1)$-brane is $1 /(2 \pi)$; using this we get,

$$
\mathcal{T}_{0}=2 \pi \mathcal{T}_{1}
$$

This gives

$$
r \equiv \frac{E_{\text {lump }}}{\mathcal{T}_{0}}=2 \pi^{2} R\left(\mathcal{V}\left(\vec{T}_{\text {lump }}\right)-\mathcal{V}\left(\vec{T}_{\text {vac }}\right)\right)
$$

The predicted answer for this ratio is 1 .

This prediction can be tested for various values of $R$, and independently of the chosen value we must obtain unity, since the mass of a D0-brane on a circle of radius $R$ does not depend on $R$. At fixed $R$ and at any level of approximation in the level expansion it is possible to use (2.16) in two ways. We can use that $2 \pi^{2} \mathcal{V}\left(\vec{T}_{\text {vac }}\right)$ at the exact vacuum is indeed -1 and thus we check how accurately

$$
r^{(1)} \equiv R\left(2 \pi^{2} \mathcal{V}_{(M, N)}\left(\vec{T}_{l u m p}\right)+1\right)
$$

approaches unity. Here $\mathcal{V}_{(M, N)}$ is the potential calculated at the specified level of approximation. Alternatively we can use the translationally invariant vacuum that is 
obtained with the same level of approximation used to compute the lump. ${ }^{8}$ This gives

$$
r^{(2)} \equiv R\left(2 \pi^{2} \mathcal{V}_{(M, N)}\left(\vec{T}_{\text {lump }}\right)-2 \pi^{2} \mathcal{V}_{(M, N)}\left(\vec{T}_{\text {vac }}\right)\right)
$$

We will find that $r^{(1)}$ approaches unity monotonically from above as we increase the level of approximation. On the other hand $r^{(2)}$ provides a more accurate answer.

\subsection{Setup and Sample Computations}

Let us now describe explicitly the string field we will be using to analyze the bosonic string lump. The zero momentum tachyon $\left|T_{0}\right\rangle=c_{1}|0\rangle$ now becomes the lowest in a family of states

$$
\left|T_{n}\right\rangle=c_{1} \cos \left(\frac{n}{R} X(0)\right)|0\rangle, \quad l\left(T_{n}\right)=\frac{n^{2}}{R^{2}}
$$

where $l\left(T_{n}\right)$ denotes the level of $T_{n}$. For any given computation only a finite number of tachyon modes are required. In the zero-momentum computation the next modes that contribute are $\left|U_{0}\right\rangle=c_{-1}|0\rangle$ and $\left|V_{0}\right\rangle=L_{-2}^{\text {matt }}|0\rangle$. In view of our remarks around (2.9) these states actually give rise to three towers

$$
\begin{aligned}
\left|U_{n}\right\rangle=c_{-1} \cos \left(\frac{n}{R} X(0)\right)|0\rangle, \quad l\left(U_{n}\right)=2+\frac{n^{2}}{R^{2}}, \\
\left|V_{n}\right\rangle=c_{1} L_{-2}^{X} \cos \left(\frac{n}{R} X(0)\right)|0\rangle, \quad l\left(V_{n}\right)=2+\frac{n^{2}}{R^{2}}, \\
\left|W_{n}\right\rangle=c_{1} L_{-2}^{\prime} \cos \left(\frac{n}{R} X(0)\right)|0\rangle, \quad l\left(W_{n}\right)=2+\frac{n^{2}}{R^{2}} .
\end{aligned}
$$

In addition to these three towers there is one more, where the $n=0$ state happens to vanish:

$$
\left|Z_{n}\right\rangle=c_{1} L_{-1}^{X} L_{-1}^{X} \cos \left(\frac{n}{R} X(0)\right)|0\rangle, \quad n \geq 1, \quad l\left(Z_{n}\right)=2+\frac{n^{2}}{R^{2}} .
$$

No new fields or towers arise until level four, and for the purposes of the present paper we shall not carry computations that far. Therefore we will use the string field

$$
|\vec{T}\rangle=t_{0}\left|T_{0}\right\rangle+t_{1}\left|T_{1}\right\rangle+t_{2}\left|T_{2}\right\rangle+\cdots
$$

\footnotetext{
${ }^{8}$ The value of $\mathcal{V}_{(M, N)}\left(\vec{T}_{v a c}\right)$ can be read from refs. [9, 11.
} 


$$
\begin{aligned}
& +u_{0}\left|U_{0}\right\rangle+u_{1}\left|U_{1}\right\rangle+\cdots \\
& +v_{0}\left|V_{0}\right\rangle+v_{1}\left|V_{1}\right\rangle+\cdots \\
& +w_{0}\left|W_{0}\right\rangle+w_{1}\left|W_{1}\right\rangle+\cdots \\
& +z_{1}\left|Z_{1}\right\rangle+\cdots
\end{aligned}
$$

Which fields and which interactions must be kept for any fixed level computation depends on the chosen radius, and this will be discussed in the following sections. We conclude here with some basic comments about the evaluation of the potential (or the action) for a string field of the above type.

This is simply the evaluation of $\mathcal{V}(\vec{T})$ as given in (2.6)

$$
\mathcal{V}(\vec{T})=\frac{1}{2}\langle\vec{T}, Q \vec{T}\rangle+\frac{1}{3}\langle\vec{T}, \vec{T} * \vec{T}\rangle .
$$

We work in units where $\alpha^{\prime}=1$. The stress tensor for the compact coordinate $X$ is $T_{X}=-\frac{1}{4} \partial X \partial X$ with $X(z) X(w) \sim-2 \ln (z-w), T(z) e^{i p \cdot X(w)} \sim \frac{p^{2}}{(z-w)^{2}} e^{i p \cdot X(w)}$ and $e^{i p_{1} \cdot X(z)} e^{i p_{2} \cdot X(w)}=(z-w)^{2 p_{1} \cdot p_{2}} e^{i p_{1} \cdot X(z)+i p_{2} \cdot X(w)}$, where $z$ and $w$ are coordinates on the real line with $z>w$. With these conventions $L_{0}|p\rangle=L_{0} e^{i p \cdot X(0)}|0\rangle=p^{2}|p\rangle$. In addition, the inner product is normalized as

$$
\left\langle\frac{n}{R}\left|c_{-1} c_{0} c_{1}\right| \frac{m}{R}\right\rangle=\delta_{n, m}
$$

Consider, for example contributions from the tachyon tower to the action. By momentum conservation all kinetic terms must be diagonal. Using (2.9) we see that the contribution from $t_{n}(n \geq 1)$ to $V$ is

$$
\frac{1}{2} \frac{t_{n}}{2} \frac{t_{n}}{2}\left(\left\langle-\frac{n}{R}\left|+\left\langle\frac{n}{R}\right|\right) c_{-1} c_{0} L_{0} c_{1}\left(\left|\frac{n}{R}\right\rangle+\left|-\frac{n}{R}\right\rangle\right)\right.\right.
$$

By momentum conservation there are two cross terms that do not vanish and give identical contributions. We thus get

$$
\frac{1}{4} t_{n}^{2}\left\langle\frac{n}{R}\left|c_{-1} c_{0}\left(-1+\frac{n^{2}}{R^{2}}\right) c_{1}\right| \frac{n}{R}\right\rangle=-\frac{1}{4}\left(1-\frac{n^{2}}{R^{2}}\right) t_{n}^{2}
$$

For $t_{0}$ the normalization factor differs by a factor of two. All this together gives us that the quadratic terms are

$$
\mathcal{V}\left(t_{0}, t_{1}, t_{2}, \cdots\right)^{(2)}=-\frac{1}{2} t_{0}^{2}-\frac{1}{4} \sum_{n=1}^{\infty}\left(1-\frac{n^{2}}{R^{2}}\right) t_{n}^{2}
$$




$$
=-\frac{1}{2} t_{0}^{2}-\frac{1}{4}\left(1-\frac{1}{R^{2}}\right) t_{1}^{2}-\frac{1}{4}\left(1-\frac{4}{R^{2}}\right) t_{2}^{2}+\cdots
$$

We will use the first tachyon harmonic $t_{1}$ to drive the unstable vacuum into the lump solution. Note that $t_{1}$ is tachyonic whenever $R>1$. We will choose different values of $R>1$ to examine how the lump forms. As $R$ increases, more and more tachyon harmonics become tachyonic.

It is not difficult to compute the interactions of the various tachyon harmonics. One can use the oscillator expressions for the states and contract them against the 3-string vertex bra $\left\langle V_{123}\right|$ [32]. Alternatively one can use the conformal field theory definition [33]

$$
\langle\vec{T}, \vec{T} * \vec{T}\rangle \equiv\left\langle h_{1} \circ T(0) h_{2} \circ T(0) h_{3} \circ T(0)\right\rangle .
$$

where $T(0)$ denotes the vertex operator associated to the state $|\vec{T}\rangle$. Here $h_{1}, h_{2}$ and $h_{3}$ are a set of familiar conformal transformations reviewed in [29]. For illustration purposes consider three tachyon harmonics $t_{n}, t_{m}$ and $t_{n+m}$, with $n \neq m \neq 0$. Such fields contribute to $\mathcal{V}$ the following interaction

$$
\frac{1}{3} \cdot 6 \cdot \frac{t_{n}}{2} \cdot \frac{t_{m}}{2} \cdot \frac{t_{n+m}}{2} \cdot 2\left\langle h_{1} \circ\left(c e^{\frac{i n X}{R}}\right)(0) h_{2} \circ\left(c e^{\frac{i m X}{R}}\right)(0) h_{3} \circ\left(c e^{\frac{-i(n+m) X}{R}}\right)(0)\right\rangle
$$

The factor $(1 / 3)$ is in the definition of $\mathcal{V}$. The factor of 6 appears because this is the number of ways three different fields can be assigned to the three punctures in the disk. Then come the fields, and then a factor of two, as there are two momentum conserving combinations giving equal contributions. Evaluation of the above gives

$$
\frac{1}{2} t_{n} t_{m} t_{n+m} K^{3-\frac{1}{R^{2}}\left(n^{2}+m^{2}+(n+m)^{2}\right)}, \quad K \equiv \frac{3 \sqrt{3}}{4}
$$

Slightly different combinatorics are required for terms of the form $t_{0} t_{n}^{2}$ and $t_{n}^{2} t_{2 n}$. Combining all such terms together we obtain

$$
\begin{aligned}
\mathcal{V}\left(t_{0}, t_{1}, \cdots\right)^{(3)}= & \frac{1}{3} K^{3} t_{0}^{3}+\frac{1}{2} \sum_{n=1}^{\infty} t_{0} t_{n}^{2} K^{3-\frac{2 n^{2}}{R^{2}}}+\frac{1}{4} \sum_{n=1}^{\infty} t_{n}^{2} t_{2 n} K^{3-\frac{6 n^{2}}{R^{2}}} \\
& +\frac{1}{2} \sum_{n \geq 1}^{\infty} \sum_{m>n}^{\infty} t_{n} t_{m} t_{n+m} K^{3-\frac{2}{R^{2}}\left(n^{2}+m^{2}+n m\right)}
\end{aligned}
$$

Equations (2.27) and (2.31) give the complete potential for the tachyon tower. 


\begin{tabular}{|l|l|}
\hline Level & Fields \\
\hline \hline 0 & $t_{0}$ \\
\hline $1 / 3$ & $t_{1}$ \\
\hline $4 / 3$ & $t_{2}$ \\
\hline 2 & $u_{0}, v_{0}, w_{0}$ \\
\hline $7 / 3$ & $u_{1}, v_{1}, w_{1}, z_{1}$ \\
\hline 3 & $t_{3}$ \\
\hline
\end{tabular}

Table 1: The list of fields appearing at various levels when $R=\sqrt{3}$.

\section{Calculating the action in the Level Expansion for $R=\sqrt{3}$}

In this section we will consider different truncation levels to calculate the lump tension. For this we will write explicitly the action at different levels. Though we will work with a fixed radius $R=\sqrt{3}$, all our equations will contain $R$ as a variable for further use. Once we know the action we can solve the equations of motion numerically for the one-lump solution by giving a nonzero initial value to $t_{1}$. At the end of the section, we will be able to study the convergence of our level truncation scheme by using both (2.17) and (2.18).

We will do these calculations at levels $(1 / 3,2 / 3),(4 / 3,8 / 3),(2,4),(7 / 3,14,3)$ and $(3,6)$. This will require the fields listed in Table [ 1 with their respective levels (using (2.19), (2.20) and (2.21)). In order to study the truncation method at various levels, we define $V(m, n)$ to be the part of the whole potential satisfying the three following conditions:

1. All terms in $V(m, n)$ have level $n$.

2. All terms in $V(m, n)$ contain only fields of level smaller than or equal to $m$. 
3. All terms in $V(m, n)$ contain at least one field of level $m$.

This definition ensures that various $V(m, n)$ 's are disjoint (i.e. $V(m, n)$ and $V\left(m^{\prime}, n^{\prime}\right)$ don't contain common terms for $\left.(m, n) \neq\left(m^{\prime}, n^{\prime}\right)\right)$. It now follows that the total potential at level $(M, N)$ is given by

$$
\mathcal{V}_{(M, N)}=\sum_{m \leq M} \sum_{n \leq N} V(m, n)
$$

We shall now compute $V(m, n)$ for $m \leq 3$ and $n \leq 6$. Though here we will restrict ourselves to levels $(M, N)$ of the form $(M, 2 M)$, eq.(3.1) and the results for $V(m, n)$ given below can be used to construct the potential $\mathcal{V}_{(M, N)}$ for arbitrary level $(M, N)$ as long as $M \leq 3$ and $N \leq 6$. We shall first list all possible terms appearing in each $V(m, n)$ consistent with momentum conservation, separating the quadratic and cubic terms. We then use the methods described in section 2 to explicitly calculate the coefficients of each possible term in the $V(m, n)$ 's.

The list of interactions that must be computed is generated conveniently with the help of the following function:

$$
\begin{aligned}
\mathcal{Z}(x, y, s) \equiv \prod_{n=0}^{\infty}\{ & \left(1-t_{n} x\left(y^{n}+y^{-n}\right) s^{n^{2} / R^{2}}\right)\left(1-u_{n} x\left(y^{n}+y^{-n}\right) s^{2+n^{2} / R^{2}}\right) \\
& \left(1-v_{n} x\left(y^{n}+y^{-n}\right) s^{2+n^{2} / R^{2}}\right)\left(1-w_{n} x\left(y^{n}+y^{-n}\right) s^{2+n^{2} / R^{2}}\right) \\
& \left.\left(1-z_{n+1} x\left(y^{n+1}+y^{-n-1}\right) s^{2+(n+1)^{2} / R^{2}}\right) \cdots\right\}^{-1}
\end{aligned}
$$

Here the formal variables $x, y$ and $s$ are used to count number of fields, momentum, and level, respectively. If we write

$$
\mathcal{Z}(x, y, s)=\sum_{m, n} \mathcal{Z}(m, n, s) x^{m} y^{n}
$$

The momentum conserving cubic interactions appear in $\mathcal{Z}(3,0, s)$ and an expansion in $s$ gives

$$
\mathcal{Z}(3,0, s)=\sum_{l} \mathcal{Z}(l) s^{l}
$$

Let $\left\{\psi^{i}\right\}$ denote the complete set of modes $\left(t_{n}, u_{n}, \cdots\right)$. Then $\mathcal{Z}(l)$ has an expression of the form $\mathcal{Z}(l) \sim \sum a_{i j k} \psi^{i} \psi^{j} \psi^{k}$ where each $a_{i j k}$ is an integer. If $a_{i j k} \neq 0$ the interaction $\psi^{i} \psi^{j} \psi^{k}$ must be included in the level $l$ contribution to the potential. Thus $\mathcal{Z}(l)$ supplies 
the complete list of momentum conserving cubic interactions of level $l$. When useful, we split by hand the terms in $\mathcal{Z}(l)$ to obtain the possible terms which appear in various $V(m, l)$ 's.

The list of all terms for the various $V(m, n)$ 's with $n \leq 6$ (and $R=\sqrt{3}$ ) are given in Table 2.

\subsection{The terms in the potential}

The explicit interactions corresponding to the various terms appearing in the table will be listed here. With $K=\frac{3 \sqrt{3}}{4}$, as in eq. 2.30 ), we have at the lowest level:

$$
V(0,0)=-\frac{1}{2} t_{0}^{2}+\frac{1}{3} K^{3} t_{0}^{3}
$$

At first nontrivial level we have:

$$
V(1 / 3,2 / 3)=-\frac{1}{4}\left(1-\frac{1}{R^{2}}\right) t_{1}^{2}+\frac{1}{2} K^{3-2 / R^{2}} t_{0} t_{1}^{2}
$$

At level 2 we have:

$$
\begin{aligned}
V(4 / 3,2) & =\frac{1}{4} K^{3-6 / R^{2}} t_{1}^{2} t_{2} \\
V(2,2) & =\frac{K}{32} t_{0}^{2}\left(22 u_{0}-5\left(v_{0}+25 w_{0}\right)\right) .
\end{aligned}
$$

At level 8/3 :

$$
\begin{aligned}
V(4 / 3,8 / 3) & =-\frac{1}{4}\left(1-\frac{4}{R^{2}}\right) t_{2}^{2}+\frac{1}{2} K^{3-8 / R^{2}} t_{0} t_{2}^{2} \\
V(2,8 / 3) & =K^{1-2 / R^{2}} t_{1}^{2}\left(\frac{11}{32} u_{0}+\frac{1}{2}\left(\frac{1}{R^{2}}-\frac{5}{32}\right) v_{0}-\frac{125}{64} w_{0}\right) \\
V(7 / 3,8 / 3) & =\frac{1}{32} K^{1-2 / R^{2}} t_{0} t_{1}\left(22 u_{1}-\left(5+\frac{16}{R^{2}}\right) v_{1}-125 w_{1}+\left(\frac{-44}{R^{2}}+\frac{32}{R^{4}}\right) z_{1}\right) .
\end{aligned}
$$

At level 4:

$$
V(2,4)=-\frac{1}{2} u_{0}^{2}+\frac{1}{4}\left(v_{0}^{2}+25 w_{0}^{2}\right)+K\left\{\frac{1}{576} t_{0}\left(76 u_{0}^{2}+179 v_{0}^{2}+9475 w_{0}^{2}\right)\right.
$$




\begin{tabular}{|l||l|l|}
\hline & Quadratic terms & Cubic terms \\
\hline \hline$V(0,0)$ & $t_{0}^{2}$ & $t_{0}^{3}$ \\
\hline \hline$V(1 / 3,2 / 3)$ & $t_{1}^{2}$ & $t_{0} t_{1}^{2}$ \\
\hline \hline$V(4 / 3,2)$ & & $t_{1}^{2} t_{2}$ \\
\hline$V(2,2)$ & & $t_{0}^{2} u_{0}, t_{0}^{2} v_{0}, t_{0}^{2} w_{0}$ \\
\hline \hline$V(4 / 3,8 / 3)$ & $t_{2}^{2}$ & $t_{0} t_{2}^{2}$ \\
\hline$V(2,8 / 3)$ & & $t_{1}^{2} u_{0}, t_{1}^{2} v_{0}, t_{1}^{2} w_{0}$ \\
\hline$V(7 / 3,8 / 3)$ & & $t_{0} t_{1} u_{1}, t_{0} t_{1} v_{1}, t_{0} t_{1} w_{1}, t_{0} t_{1} z_{1}$ \\
\hline \hline$V(2,4)$ & $u_{0}^{2}, v_{0}^{2}, w_{0}^{2}$ & $t_{0} u_{0}^{2}, t_{0} v_{0}^{2}, t_{0} w_{0}^{2}, t_{0} u_{0} v_{0}, t_{0} u_{0} w_{0}, t_{0} v_{0} w_{0}$ \\
\hline$V(7 / 3,4)$ & & $t_{1} t_{2} u_{1}, t_{1} t_{2} v_{1}, t_{1} t_{2} w_{1}, t_{1} t_{2} z_{1}$ \\
\hline \hline$V(2,14 / 3)$ & & $t_{2}^{2} u_{0}, t_{2}^{2} v_{0}, t_{2}^{2} w_{0}$ \\
\hline$V(7 / 3,14 / 3)$ & $u_{1}^{2}, v_{1}^{2}, w_{1}^{2}, z_{1}^{2}$, & $t_{0} u_{1}^{2}, t_{0} v_{1}^{2}, t_{0} w_{1}^{2}, t_{0} u_{1} v_{1}, t_{0} u_{1} w_{1}, t_{0} v_{1} w_{1}$, \\
$t_{1} u_{0} u_{1}, t_{1} u_{0} v_{1}, t_{1} u_{0} w_{1}, t_{1} v_{0} u_{1}, t_{1} v_{0} v_{1}$, \\
$t_{1} v_{0} w_{1}, t_{1} w_{0} u_{1}, t_{1} w_{0} v_{1}, t_{1} w_{0} w_{1}$, \\
$t_{0} z_{1}^{2}, t_{0} u_{1} z_{1}, t_{0} v_{1} z_{1}, t_{0} w_{1} z_{1}$, \\
$t_{1} u_{0} z_{1}, t_{1} v_{0} z_{1}, t_{1} w_{0} z_{1}$ \\
\hline$V(7 / 3,6)$ & & $t_{1} t_{2} t_{3}$ \\
\hline \hline$V(3,14 / 3)$ & & $u_{0}^{3}, v_{0}^{3}, w_{0}^{3}, u_{0}^{2} v_{0}, u_{0}^{2} w_{0}, u_{0} v_{0}^{2}, u_{0} w_{0}^{2}, v_{0}^{2} w_{0}$ \\
$v_{0} w_{0}^{2}, u_{0} v_{0} w_{0}$, \\
\hline
\end{tabular}

Table 2: Quadratic terms and interactions appearing at various levels when $R=\sqrt{3}$. 


$$
\begin{gathered}
\left.+\frac{625}{864} t_{0} v_{0} w_{0}-\frac{55}{432} t_{0} u_{0}\left(v_{0}+25 w_{0}\right)\right\} \\
V(7 / 3,4)=\frac{1}{64} K^{1-6 / R^{2}} t_{1} t_{2}\left(22 u_{1}-\left(5-\frac{48}{R^{2}}\right) v_{1}-125 w_{1}+\left(-\frac{44}{R^{2}}+\frac{288}{R^{4}}\right) z_{1}\right) .
\end{gathered}
$$

At level 14/3:

$$
\begin{aligned}
& V(2,14 / 3)=\frac{1}{64} K^{1-8 / R^{2}} t_{2}^{2}\left(22 u_{0}-\left(5-\frac{128}{R^{2}}\right) v_{0}-125 w_{0}\right) \\
& V\left(\frac{7}{3}, \frac{14}{3}\right)=\frac{1}{8}\left(1+\frac{1}{R^{2}}\right)\left(-2 u_{1}^{2}+\left(1+\frac{8}{R^{2}}\right) v_{1}^{2}+25 w_{1}^{2}+\left(\frac{8}{R^{2}}+\frac{16}{R^{4}}\right) z_{1}^{2}+\frac{24}{R^{2}} v_{1} z_{1}\right) \\
& +K^{1-2 / R^{2}}\left\{\frac{19}{288} t_{0} u_{1}^{2}+\frac{1}{3456}\left(537+\frac{8864}{R^{2}}+\frac{256}{R^{4}}\right) t_{0} v_{1}^{2}+\frac{28425}{3456} t_{0} w_{1}^{2}\right. \\
& -\frac{11}{864} t_{0} u_{1}\left(\left(5+\frac{16}{R^{2}}\right) v_{1}+125 w_{1}\right)+\frac{125}{1728}\left(5+\frac{16}{R^{2}}\right) t_{0} v_{1} w_{1} \\
& +\frac{19}{144} t_{1} u_{0} u_{1}-\frac{11}{864} t_{1} u_{0}\left(\left(5+\frac{16}{R^{2}}\right) v_{1}+125 w_{1}\right) \\
& -\frac{11}{864}\left(5-\frac{32}{R^{2}}\right) t_{1} v_{0} u_{1}+\frac{1}{1728}\left(537+\frac{944}{R^{2}}-\frac{512}{R^{4}}\right) t_{1} v_{0} v_{1} \\
& +\frac{25}{1728}\left(25-\frac{160}{R^{2}}\right) t_{1} v_{0} w_{1}-\frac{1375}{864} t_{1} w_{0} u_{1}+\frac{25}{1728}\left(25+\frac{80}{R^{2}}\right) t_{1} w_{0} v_{1} \\
& +\frac{28425}{1728} t_{1} w_{0} w_{1}+\frac{1}{216} \frac{1}{R^{2}}\left(384+\frac{1145}{R^{2}}+\frac{336}{R^{4}}+\frac{64}{R^{6}}\right) t_{0} z_{1}^{2} \\
& +\frac{11}{864} \frac{1}{R^{2}}\left(-44+\frac{32}{R^{2}}\right) t_{0} u_{1} z_{1}+\frac{1}{432}\left(\frac{2359}{R^{2}}+\frac{1672}{R^{4}}-\frac{128}{R^{6}}\right) t_{0} v_{1} z_{1} \\
& +\frac{125}{432} \frac{1}{R^{2}}\left(11-\frac{8}{R^{2}}\right)\left(t_{0} w_{1} z_{1}+t_{1} w_{0} z_{1}\right) \\
& \left.+\frac{1}{864} \frac{1}{R^{2}}\left(11\left(-44+\frac{32}{R^{2}}\right) t_{1} u_{0} z_{1}+\left(2158-\frac{2832}{R^{2}}+\frac{512}{R^{4}}\right) t_{1} v_{0} z_{1}\right)\right\} \\
& V(3,14 / 3)=\frac{1}{2} K^{3-14 / R^{2}} t_{1} t_{2} t_{3}
\end{aligned}
$$


And at level 6:

$$
\begin{aligned}
V(2,6)= & K\left\{\frac{1}{144} u_{0}^{3}+\frac{8321}{93312} v_{0}^{3}-\frac{219775}{10368} w_{0}^{3}-\frac{95}{7776} u_{0}^{2}\left(v_{0}+25 w_{0}\right)\right. \\
& \left.+\frac{1969}{15552} u_{0} v_{0}^{2}+\frac{104225}{15552} u_{0} w_{0}^{2}-\frac{22375}{31104} v_{0}^{2} w_{0}-\frac{47375}{31104} v_{0} w_{0}^{2}+\frac{6875}{23328} u_{0} v_{0} w_{0}\right\} \\
V(7 / 3,6)= & K^{1-6 / R^{2}}\left\{\frac{19}{576} t_{2} u_{1}^{2}+\frac{1}{2304}\left(179-\frac{1696}{R^{2}}+\frac{768}{R^{4}}\right) t_{2} v_{1}^{2}+\frac{9475}{2304} t_{2} w_{1}^{2}\right. \\
& -\frac{11}{1728}\left(5-\frac{48}{R^{2}}\right) t_{2} u_{1} v_{1}-\frac{1375}{1728} t_{2} u_{1} w_{1}+\frac{1}{72}\left(\frac{625}{48}-\frac{125}{R^{2}}\right) t_{2} v_{1} w_{1} \\
& +\frac{1}{144}\left(-\frac{128}{R^{2}}+\frac{723}{R^{4}}-\frac{2064}{R^{6}}+\frac{1728}{R^{8}}\right) t_{2} z_{1}^{2}+\frac{11}{432}\left(\frac{-11}{R^{2}}+\frac{72}{R^{4}}\right) t_{2} u_{1} z_{1} \\
& \left.+\frac{1}{288}\left(-\frac{67}{R^{2}}-\frac{808}{R^{4}}+\frac{1152}{R^{6}}\right) t_{2} v_{1} z_{1}+\frac{125}{864}\left(\frac{11}{R^{2}}-\frac{72}{R^{4}}\right) t_{2} w_{1} z_{1}\right\} \\
V(3,6)= & \frac{1}{4}\left(-1+\frac{9}{R^{2}}\right) t_{3}^{2}+\frac{1}{2} K^{3-18 / R^{2}} t_{0} t_{3}^{2} .
\end{aligned}
$$

\subsection{Potentials at various truncation levels and mass calcula- tions}

From these formulae one can construct the potentials at various truncation levels using (3.1). As we will use them, we give below the explicit sums for $\mathcal{V}_{(1 / 3,2 / 3)}, \mathcal{V}_{(4 / 3,8 / 3)}$, $\mathcal{V}_{(2,4)}, \mathcal{V}_{(7 / 3,14 / 3)}$ and $\mathcal{V}_{(3,6)}$ :

$$
\begin{aligned}
\mathcal{V}_{(1 / 3,2 / 3)} & =V(0,0)+V(1 / 3,2 / 3) \\
\mathcal{V}_{(4 / 3,8 / 3)} & =\mathcal{V}_{(1 / 3,2 / 3)}+V(4 / 3,2)+V(4 / 3,8 / 3) \\
\mathcal{V}_{(2,4)} & =\mathcal{V}_{(4 / 3,8 / 3)}+V(2,2)+V(2,8 / 3)+V(2,4) \\
\mathcal{V}_{(7 / 3,14 / 3)} & =\mathcal{V}_{(2,4)}+V(7 / 3,8 / 3)+V(7 / 3,4)+V(2,14 / 3)+V(7 / 3,14 / 3) \\
\mathcal{V}_{(3,6)} & =\mathcal{V}_{(7 / 3,14 / 3)}+V(3,14 / 3)+V(2,6)+V(7 / 3,6)+V(3,6)
\end{aligned}
$$

In general, the potential at a given level has many extrema. Two of them will be of particular interest for us:

1. We always find a translationally invariant minimum $\vec{T}_{v a c}$ corresponding to the tachyon condensation. At this minimum, all fields with nonzero momentum have 


\begin{tabular}{|c|c|c|c|c|c|}
\hline Field & $(1 / 3,2 / 3)$ & $(4 / 3,8 / 3)$ & $(2,4)$ & $(7 / 3,14 / 3)$ & $(3,6)$ \\
\hline \hline$t_{0}$ & 0.181034 & 0.214757 & 0.25703 & 0.265131 & 0.269224 \\
\hline$t_{1}$ & -0.344389 & -0.343566 & -0.384575 & -0.394396 & -0.394969 \\
\hline$t_{2}$ & $\ldots$ & -0.0955972 & -0.107424 & -0.12046 & -0.125011 \\
\hline$u_{0}$ & $\ldots$ & $\ldots$ & 0.0888087 & 0.0900609 & 0.0969175 \\
\hline$v_{0}$ & $\ldots$ & $\ldots$ & -0.00675676 & -0.0175367 & -0.0172906 \\
\hline$w_{0}$ & $\ldots$ & $\ldots$ & 0.0317837 & 0.0299617 & 0.0320394 \\
\hline$u_{1}$ & $\ldots$ & $\ldots$ & $\ldots$ & -0.0643958 & -0.0648543 \\
\hline$v_{1}$ & $\ldots$ & $\ldots$ & $\ldots$ & 0.0540447 & 0.0505836 \\
\hline$w_{1}$ & $\ldots$ & $\ldots$ & $\ldots$ & -0.0187778 & -0.0189058 \\
\hline$z_{1}$ & $\ldots$ & $\ldots$ & $\ldots$ & -0.0698363 & -0.0665402 \\
\hline$t_{3}$ & $\ldots$ & $\ldots$ & $\ldots$ & $\ldots$ & -0.0142169 \\
\hline
\end{tabular}

Table 3: The values of various modes of the string field at the stationary point of the potential for $R=\sqrt{3}$ calculated at various levels of approximation.

zero vev. We will use this solution when calculating the ratio $r^{(2)}$ defined in eq.(2.18).

2. If we start the numerical algorithm with initial values near $t_{0} \approx 0.25$ and $t_{1} \approx$ -0.4 then our numerical algorithm converges to the one-lump solution $\vec{T}_{\text {lump }}$ that we are interested in.

The solution $\vec{T}_{\text {vac }}$ can be found in refs. [9, 11]. In table [3 we give the solutions $\vec{T}_{\text {lump }}$ at various truncation levels. Having found $\vec{T}_{\text {vac }}$ and $\vec{T}_{\text {lump }}$ we can now calculate the ratio of the lump mass to the D0-brane mass using the two different methods (2.17) and (2.18). The results are given in Table 6 . We see that the first method gives a monotonically decreasing lump mass whereas the second method is oscillating but 


\begin{tabular}{|l||l|l|}
\hline Level & $r^{(1)}$ & $r^{(2)}$ \\
\hline \hline$(1 / 3 ; 2 / 3)$ & 1.32002 & 0.77377 \\
\hline$(4 / 3 ; 8 / 3)$ & 1.25373 & 0.707471 \\
\hline$(2 ; 4)$ & 1.11278 & 1.02368 \\
\hline$(7 / 3 ; 14 / 3)$ & 1.07358 & 0.984467 \\
\hline$(3,6)$ & 1.06421 & 0.993855 \\
\hline
\end{tabular}

Table 4: The ratio of the calculated mass of the lump to the mass of the D0 brane in the two schemes described in equations (2.17) and (2.18).

gives a lump mass much closer to the expected mass.

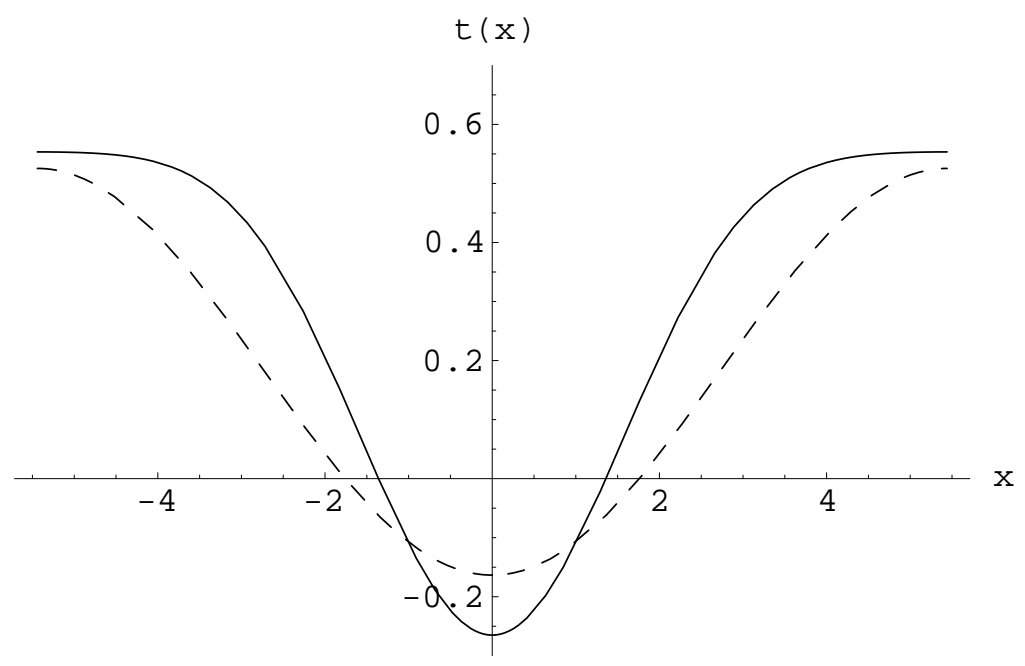

Figure 1: The dashed line shows a plot of $t(x)$ for $R=\sqrt{3}$ at level $(1 / 3,2 / 3)$ approximation. The solid line shows the plot of $t(x)$ for $R=\sqrt{3}$ at the level $(3,6)$ approximation. 


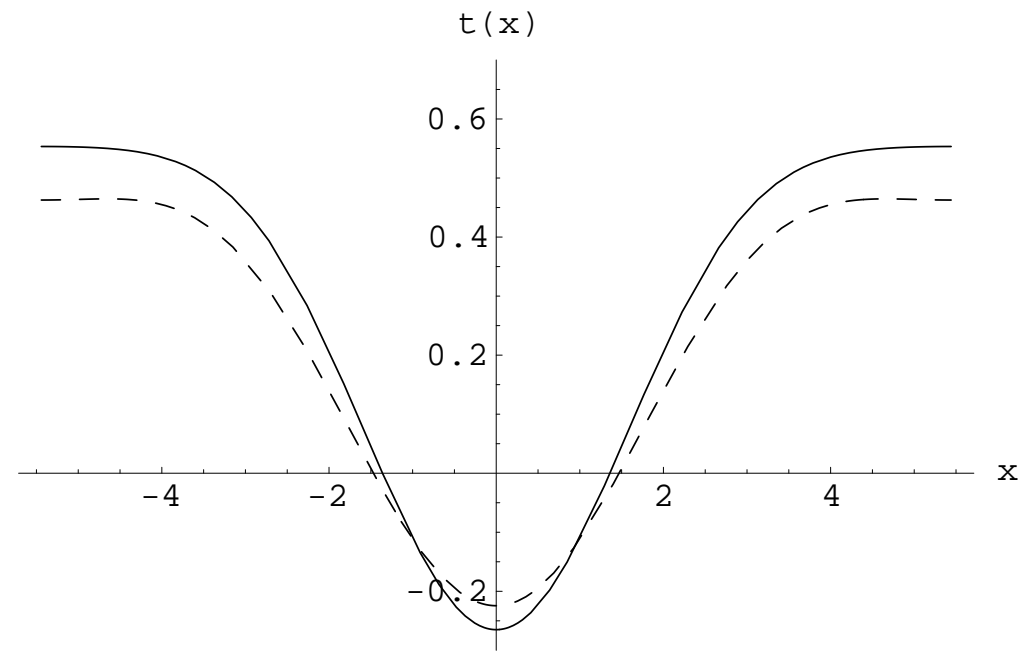

Figure 2: The dashed line shows a plot of $t(x)$ for $R=\sqrt{3}$ at level $(4 / 3,8 / 3)$ approximation. The solid line shows the plot of $t(x)$ for $R=\sqrt{3}$ at the level $(3,6)$ approximation.

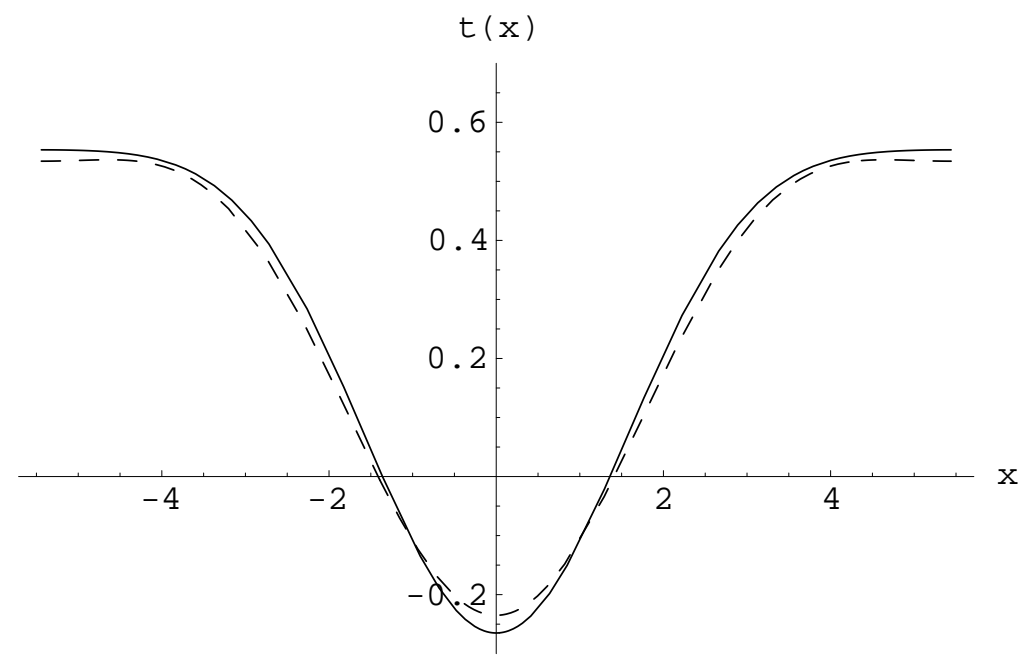

Figure 3: The dashed line shows a plot of $t(x)$ for $R=\sqrt{3}$ at level $(2,4)$ approximation. The solid line shows the plot of $t(x)$ for $R=\sqrt{3}$ at the level $(3,6)$ approximation. 


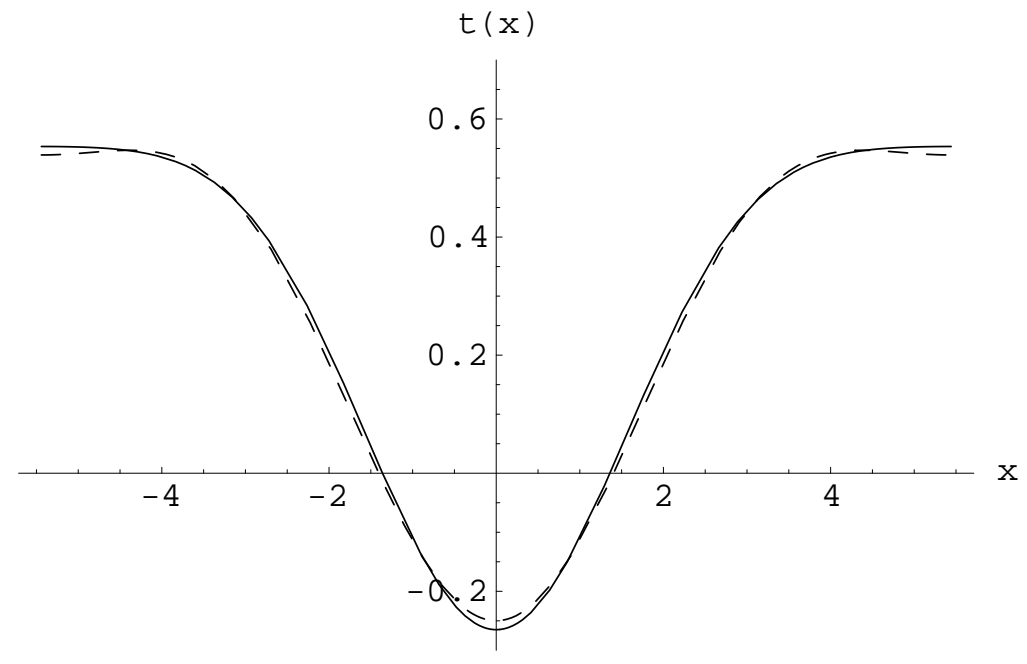

Figure 4: The dashed line shows a plot of $t(x)$ for $R=\sqrt{3}$ at level $(7 / 3,14 / 3)$ approximation. The solid line shows the plot of $t(x)$ for $R=\sqrt{3}$ at the level $(3,6)$ approximation.

It is instructive to plot the profile of the tachyon field:

$$
t(x)=\sum_{n} t_{n} \cos \frac{n x}{R}
$$

as a function of $x$ and compare them at different approximations. In figs. 1 t we have plotted the tachyon profiles at the level $(1 / 3,2 / 3),(4 / 3,8 / 3),(2,4)$ and $(7 / 3,14 / 3)$ approximation respectively, each of them being superimposed on the tachyon profile at the level $(3,6)$ approximation.

For future use, we shall now define two new functions $F_{0}$ and $G_{0}$ as follows:

$$
\begin{aligned}
F_{0}\left(t_{0}, t_{1}, t_{2}, u_{0}, v_{0}, w_{0}, u_{1}, v_{1}, w_{1}, z_{1} ; R\right)= & \mathcal{V}_{\left(\frac{7}{3}, \frac{14}{3}\right)} \\
G_{0}\left(t_{0}, t_{1}, t_{2}, u_{0}, v_{0}, w_{0}, u_{1}, v_{1}, w_{1}, z_{1} ; R\right)= & \mathcal{V}_{(3,6)}-V(3,6)-V(2,14 / 3)-V(7 / 3,4) \\
& -V(7 / 3,6)-V(3,14 / 3)
\end{aligned}
$$

where $\mathcal{V}_{\left(\frac{7}{3}, \frac{14}{3}\right)}$ and $\mathcal{V}_{(3,6)}$ have been defined in eqs.(3.5)-(3.12). The right hand side of this equation has to be interpreted as a function of the various modes $t_{0}, \ldots z_{1}$ and $R$, without $R$ being set to $\sqrt{3}$. The function $F_{0}$ and $G_{0}$ defined here will be useful in 
constructing the potential $\mathcal{V}_{(M, N)}$ for other values of $R$, as will be discussed in the next section.

\section{Tachyon Lump at Other Radii}

In this section we shall discuss the construction of the tachyonic lump solution on circles of radii other than $\sqrt{3}$, and compare the results with those obtained for $R=\sqrt{3}$. As the basic techniques have already been discussed in the previous two sections, in this section we shall only quote the results.

\section{1 $R>\sqrt{3}$}

First we need to decide which values of $R$ we shall use to study the lump. Although this choice is arbitrary, there is slight simplification of counting levels if we choose $R$ such that the level of $u_{1}, v_{1}, w_{1}$ and $z_{1}$ coincide with that of one of the harmonics (say $t_{n}$ ) of the tachyon field. This requires

$$
2+\frac{1}{R^{2}}=\frac{n^{2}}{R^{2}}, \quad \rightarrow \quad R=\sqrt{\frac{n^{2}-1}{2}} .
$$

We shall consider the values $n=4,5,6$ corresponding to $R=\sqrt{\frac{15}{2}}, \sqrt{12}, \sqrt{\frac{35}{2}}$. In each case we shall be using the level $\left(2+\frac{1}{R^{2}}, 4+\frac{2}{R^{2}}\right)$ approximation to the potential. For this we need to include up to the $n$-th harmonic of the tachyon field $t$ and the first harmonics of the fields $u, v, w$ and $z$.

For these additional $R$ values all interactions present in $\mathcal{V}_{\left(\frac{7}{3}, \frac{14}{3}\right)}$ at $R=\sqrt{3}$ are still present. We need, however, further interactions as can be checked using the generating function (3.2). These additional interactions can be expressed in terms of the following functions:

$$
\begin{aligned}
& F_{1}\left(t_{0}, \cdots, t_{4}, u_{0}, v_{0}, w_{0}, u_{1}, v_{1}, w_{1}, z_{1} ; R\right) \\
= & -\frac{1}{4}\left(1-\frac{9}{R^{2}}\right) t_{3}^{2}-\frac{1}{4}\left(1-\frac{16}{R^{2}}\right) t_{4}^{2} \\
+ & \frac{1}{2} K^{3-18 / R^{2}} t_{0} t_{3}^{2}+\frac{1}{2} K^{3-32 / R^{2}} t_{0} t_{4}^{2}+\frac{1}{2} K^{3-14 / R^{2}} t_{1} t_{2} t_{3}+\frac{1}{4} K^{3-24 / R^{2}} t_{2}^{2} t_{4}+\frac{1}{2} K^{3-26 / R^{2}} t_{1} t_{3} t_{4} \\
+ & \left(\frac{11}{32} u_{1}-\frac{125}{64} w_{1}+\left(\frac{25}{2 R^{4}}-\frac{11}{16 R^{2}}\right) z_{1}+\left(\frac{11}{4 R^{2}}-\frac{5}{64}\right) v_{1}\right) K^{1-14 / R^{2}} t_{2} t_{3}
\end{aligned}
$$




$$
\begin{aligned}
& F_{2}\left(t_{0}, \cdots, t_{5}, u_{0}, v_{0}, w_{0}, u_{1}, v_{1}, w_{1}, z_{1} ; R\right) \\
= & -\frac{1}{4}\left(1-\frac{25}{R^{2}}\right) t_{5}^{2}+\frac{1}{2} K^{3-38 / R^{2}} t_{2} t_{3} t_{5}+\frac{1}{2} K^{3-42 / R^{2}} t_{1} t_{4} t_{5}+\frac{1}{2} K^{3-50 / R^{2}} t_{0} t_{5}^{2} \\
& +\left(\frac{11}{32} u_{0}+\left(-\frac{5}{64}+\frac{9}{2 R^{2}}\right) v_{0}-\frac{125}{64} w_{0}\right) K^{1-18 / R^{2}} t_{3}^{2} \\
& +\left(\frac{11}{32} u_{1}+\left(-\frac{5}{64}+\frac{23}{4 R^{2}}\right) v_{1}-\frac{125}{64} w_{1}+\left(\frac{49}{2 R^{4}}-\frac{11}{16 R^{2}}\right) z_{1}\right) K^{1-26 / R^{2}} t_{3} t_{4} \\
& F_{3}\left(t_{0}, \cdots, t_{6}, u_{0}, v_{0}, w_{0}, u_{1}, v_{1}, w_{1}, z_{1} ; R\right) \\
= & -\frac{1}{4}\left(1-\frac{36}{R^{2}}\right) t_{6}^{2}+\frac{1}{4} K^{3-54 / R^{2}} t_{3}^{2} t_{6}+\frac{1}{2} K^{3-56 / R^{2}} t_{2} t_{4} t_{6}+\frac{1}{2} K^{3-62 / R^{2}} t_{1} t_{5} t_{6} \\
& +\frac{1}{2} K^{3-72 / R^{2}} t_{0} t_{6}^{2}+\left(\frac{11}{32} u_{0}+\left(-\frac{5}{64}+\frac{8}{R^{2}}\right) v_{0}-\frac{125}{64} w_{0}\right) K^{1-32 / R^{2}} t_{4}^{2} .
\end{aligned}
$$

We shall now write down our results for level $\left(2+\frac{1}{R^{2}}, 4+\frac{2}{R^{2}}\right)$ approximation for the potential for $R^{2}=\left(n^{2}-1\right) / 2$ in terms of the functions $F_{0}, \ldots F_{3}$ defined in eqs.(3.14), (4.2)-(4.4). These are as follows:

$$
\begin{aligned}
& \mathcal{V}_{(32 / 15,64 / 15)}\left(t_{0}, \cdots, t_{4}, u_{0}, v_{0}, w_{0}, u_{1}, v_{1}, w_{1}, z_{1} ; R=\sqrt{15 / 2}\right) \\
&= F_{0}\left(t_{0}, t_{1}, t_{2}, u_{0}, v_{0}, w_{0}, u_{1}, v_{1}, w_{1}, z_{1} ; R=\sqrt{15 / 2}\right) \\
&+ F_{1}\left(t_{0}, \cdots, t_{4}, u_{0}, v_{0}, w_{0}, u_{1}, v_{1}, w_{1}, z_{1} ; R=\sqrt{15 / 2}\right) \\
& \mathcal{V}_{(25 / 12,25 / 6)}\left(t_{0}, \cdots, t_{5}, u_{0}, v_{0}, w_{0}, u_{1}, v_{1}, w_{1}, z_{1} ; R=\sqrt{12}\right) \\
&= F_{0}\left(t_{0}, t_{1}, t_{2}, u_{0}, v_{0}, w_{0}, u_{1}, v_{1}, w_{1}, z_{1} ; R=\sqrt{12}\right) \\
&+F_{1}\left(t_{0}, \cdots, t_{4}, u_{0}, v_{0}, w_{0}, u_{1}, v_{1}, w_{1}, z_{1} ; R=\sqrt{12}\right) \\
&+F_{2}\left(t_{0}, \cdots, t_{5}, u_{0}, v_{0}, w_{0}, u_{1}, v_{1}, w_{1}, z_{1} ; R=\sqrt{12}\right) \\
& \mathcal{V}_{(72 / 35,144 / 35)}\left(t_{0}, \cdots, t_{6}, u_{0}, v_{0}, w_{0}, u_{1}, v_{1}, w_{1}, z_{1} ; R=\sqrt{35 / 2}\right) \\
& F_{0}\left(t_{0}, t_{1}, t_{2}, u_{0}, v_{0}, w_{0}, u_{1}, v_{1}, w_{1}, z_{1} ; R=\sqrt{35 / 2}\right) \\
&+ F_{1}\left(t_{0}, \cdots, t_{4}, u_{0}, v_{0}, w_{0}, u_{1}, v_{1}, w_{1}, z_{1} ; R=\sqrt{35 / 2}\right) \\
&+ F_{2}\left(t_{0}, \cdots, t_{5}, u_{0}, v_{0}, w_{0}, u_{1}, v_{1}, w_{1}, z_{1} ; R=\sqrt{35 / 2}\right) \\
&+ F_{3}\left(t_{0}, \cdots, t_{6}, u_{0}, v_{0}, w_{0}, u_{1}, v_{1}, w_{1}, z_{1} ; R=\sqrt{35 / 2}\right) .
\end{aligned}
$$


As in the previous section, we can find a tachyonic lump solution by starting with a non-zero seed value of $t_{1}$. The numerical solutions are given in Table 5. The result for the two ratios $r^{(1)}$ and $r^{(2)}$, defined in eqs. (2.17) and (2.18) are given in Table 6 .

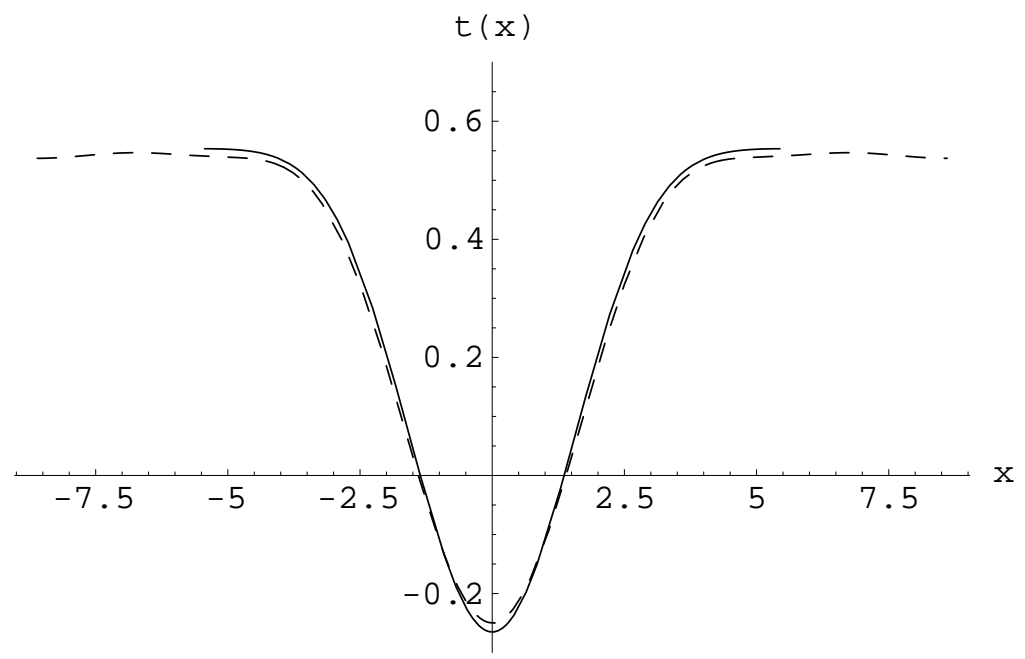

Figure 5: The dashed line shows a plot of $t(x)$ for $R=\sqrt{15 / 2}$ at level $(32 / 15,64 / 15)$ approximation. The solid line spanning a smaller range of $x$ shows the plot of $t(x)$ for the level $(3,6)$ approximation at $R=\sqrt{3}$.

In Figs.57 we have plotted the tachyon field $t(x)$ defined in eq.(3.13) as a function of $x$ for each of the three values of $R$. For reference we have also plotted on the same graph the function $t(x)$ obtained in the level $(3,6)$ approximation for $R=\sqrt{3}$. As is seen from these figures, the tachyon profiles for different radii are almost undistinguishable from each other even though they are obtained as superpositions of harmonics of very different wave-lengths.

\section{$4.2 R<\sqrt{3}$}

Finally we would like to study how the shape of the soliton changes when $R$ is small. For this we take $R=\sqrt{1.1}$ and work at the level $(40 / 11,80 / 11)$ approximation of the potential. One can show that to this level of approximation the potential is given by,

$$
\mathcal{V}_{(40 / 11,80 / 11)}\left(t_{0}, t_{1}, t_{2}, u_{0}, v_{0}, w_{0}, u_{1}, v_{1}, w_{1}, z_{1} ; R=\sqrt{1.1}\right)
$$




\begin{tabular}{|c|c|c|c|c|}
\hline Field & $R=\sqrt{15 / 2}$ & $R=\sqrt{12}$ & $R=\sqrt{35 / 2}$ & $R=\sqrt{11 / 10}$ \\
\hline \hline$t_{0}$ & 0.363333 & 0.401189 & 0.424556 & 0.0804185 \\
\hline$t_{1}$ & -0.308419 & -0.255373 & -0.218344 & -0.31707 \\
\hline$t_{2}$ & -0.19463 & -0.190921 & -0.176679 & -0.00983574 \\
\hline$t_{3}$ & -0.0849552 & -0.122721 & -0.132269 & $\ldots$ \\
\hline$t_{4}$ & -0.0248729 & -0.0575418 & -0.0830114 & $\ldots$ \\
\hline$t_{5}$ & $\ldots$ & -0.0210929 & -0.0409281 & $\ldots$ \\
\hline$t_{6}$ & $\ldots$ & $\ldots$ & -0.0178687 & $\ldots$ \\
\hline$u_{0}$ & 0.118792 & 0.131499 & 0.139048 & 0.0318155 \\
\hline$v_{0}$ & 0.0131977 & 0.020668 & 0.0263317 & -0.0591248 \\
\hline$w_{0}$ & 0.0380389 & 0.0417417 & 0.0438076 & 0.0132021 \\
\hline$u_{1}$ & -0.0712708 & -0.0629211 & -0.0567058 & -0.0052739 \\
\hline$v_{1}$ & -0.0958004 & -0.0657449 & -0.0476215 & -0.0119114 \\
\hline$w_{1}$ & -0.0181708 & -0.0150031 & -0.0131234 & -0.000863176 \\
\hline$z_{1}$ & 0.0860302 & 0.058747 & 0.0418645 & 0.00570249 \\
\hline & & & & \\
\hline
\end{tabular}

Table 5: The values of various modes of the string field at the stationary point of the potential for different radii. 


\begin{tabular}{|l||l|l|}
\hline$R$ & $r^{(1)}$ & $r^{(2)}$ \\
\hline \hline$\sqrt{15 / 2}$ & 1.14625 & 1.00535 \\
\hline$\sqrt{12}$ & 1.19147 & 1.01324 \\
\hline$\sqrt{35 / 2}$ & 1.23876 & 1.02353 \\
\hline$\sqrt{11 / 10}$ & 1.02175 & 0.979149 \\
\hline
\end{tabular}

Table 6: The ratio of the calculated mass of the lump to the mass of the D0 brane at various radii in the two schemes described in equations (2.17) and (2.18).

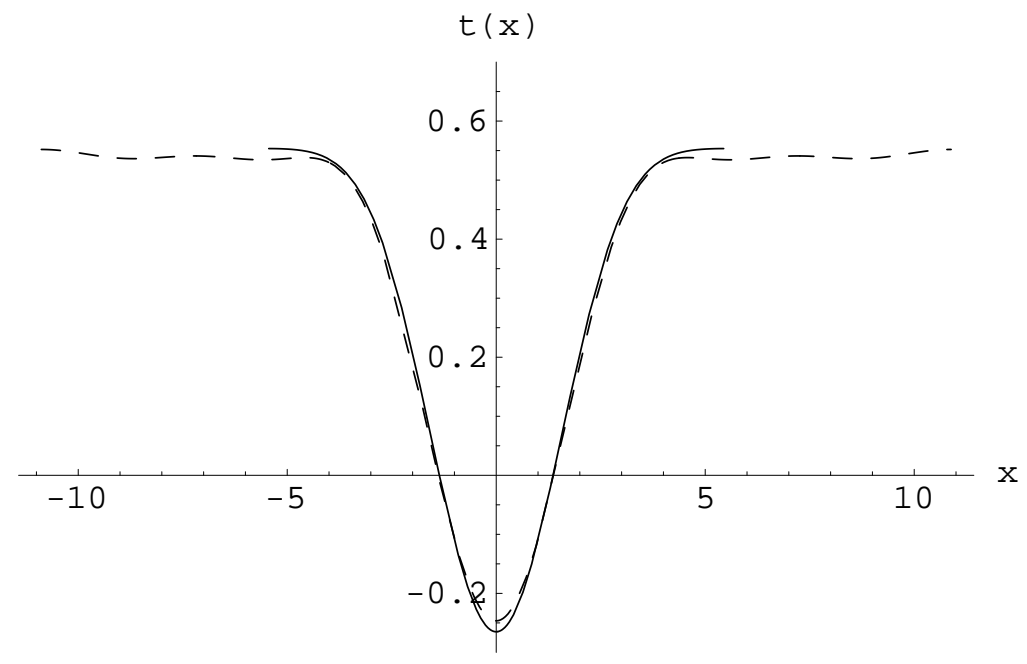

Figure 6: The dashed line shows a plot of $t(x)$ for $R=\sqrt{12}$ at level $(25 / 12,25 / 6)$ approximation. The solid line spanning a smaller range of $x$ shows the plot of $t(x)$ for the level $(3,6)$ approximation at $R=\sqrt{3}$.

$$
=G_{0}\left(t_{0}, t_{1}, t_{2}, u_{0}, v_{0}, w_{0}, u_{1}, v_{1}, w_{1}, z_{1} ; R=\sqrt{1.1}\right),
$$

where $G_{0}$ has been defined in eq.(3.14). The tachyonic lump solution for this potential is given in table 5. The results for the two ratios $r^{(1)}$ and $r^{(2)}$ defined in eqs. 2.17) and (2.18) are given in table 6 . 


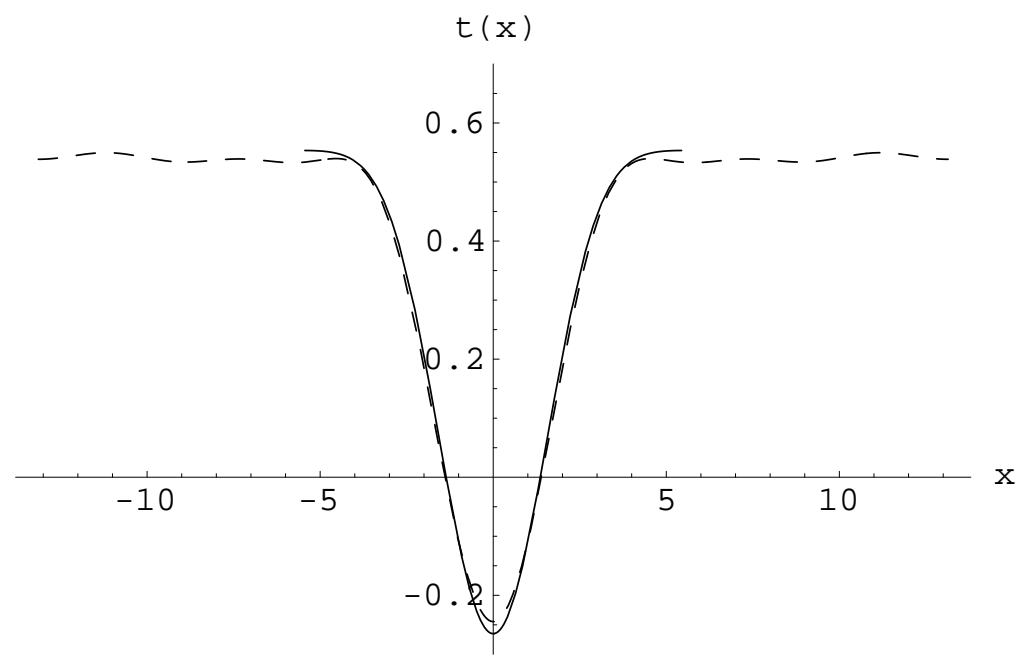

Figure 7: The dashed line shows a plot of $t(x)$ for $R=\sqrt{35 / 2}$ at level $(72 / 35,144 / 35)$ approximation. The solid line spanning a smaller range of $x$ shows the plot of $t(x)$ for the level $(3,6)$ approximation at $R=\sqrt{3}$.

We have displayed in fig. 8 the tachyon profile, superimposed on the tachyon profile for the level $(3,6)$ approximation at $R=\sqrt{3}$. As can be seen from this figure, for $R=\sqrt{11 / 10}$ there is not enough room for the full lump solution to fit in, but the profile of the lump at smaller radius follows closely the profile at larger radius near the core.

\subsection{Size of the lump}

We can estimate the size of the lump at different radii in a somewhat systematic way by fitting the lump profile with a gaussian curve of the form:

$$
G(x)=a+b \cdot e^{-x^{2} /\left(2 \sigma^{2}\right)} .
$$

We calculate the parameters $a, b$ and $\sigma$ using a nonlinear regression algorithm on a set of points chosen on the lump profile in the following way. For $R \geq \sqrt{3}$ :

- We take 100 points, regularly spaced in $x$, in the core of the lump from $x=-\sqrt{3} \pi$ to $x=\sqrt{3} \pi$. 


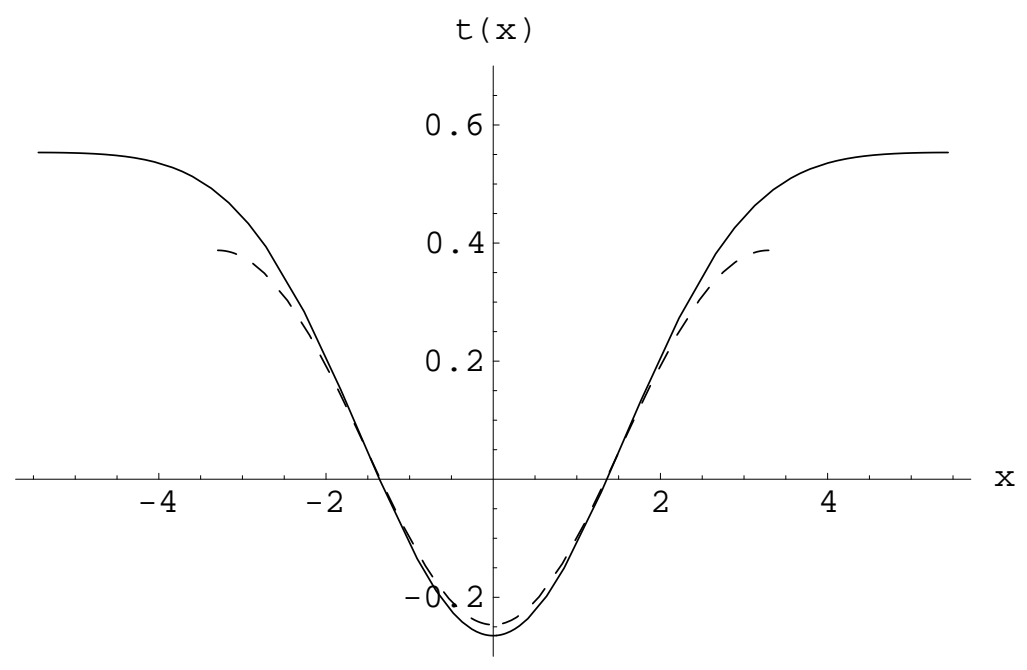

Figure 8: The dashed line shows a plot of $t(x)$ for $R=\sqrt{11 / 10}$ at level $(40 / 11,80 / 11)$ approximation. The solid line spanning a smaller range of $x$ shows the plot of $t(x)$ for the level $(3,6)$ approximation at $R=\sqrt{3}$.

- We take a smaller density of points, regularly spaced in $x$, on the rest of the circle (where the profile is essentially flat). Here we have taken 20, 30 and 40 points for $R=\sqrt{15 / 2}, R=\sqrt{12}$ and $R=\sqrt{35 / 2}$ respectively.

In the case of $R=\sqrt{11 / 10}$, we take 100 points from $x=-\sqrt{11 / 10} \pi$ to $x=\sqrt{11 / 10} \pi$

The results of the regression at the different radii are given in table 7. We see that the size of the lump, which can be defined as a multiple of $\sigma$, is essentially independent of the radius (it increases by about $1.5 \%$ when $\mathrm{R}$ increases from $\sqrt{3}$ to $\sqrt{35 / 2}$ ). Even when there is not enough room for the lump to fit in $(R=\sqrt{11 / 10})$, the lump is only slightly compressed (by about $7 \%$ ). A reasonable definition for the size would be $6 \sigma$, with the solution extending by $3 \sigma$ both along the positive and the negative $x$-axis. With this convention, the lump will have a size of approximately $9.3 \sqrt{\alpha^{\prime}}$. This is close to the answer obtained in ref. 12. 


\begin{tabular}{|c|c|c|c|}
\hline Radius & $\mathrm{a}$ & $\mathrm{b}$ & $\sigma$ \\
\hline \hline$\sqrt{3}$ & 0.559814 & -0.828599 & 1.52341 \\
\hline$\sqrt{15 / 2}$ & 0.546313 & -0.804112 & 1.5595 \\
\hline$\sqrt{12}$ & 0.544226 & -0.801652 & 1.54089 \\
\hline$\sqrt{35 / 2}$ & 0.54328 & -0.799957 & 1.54477 \\
\hline$\sqrt{11 / 10}$ & 0.451678 & -0.702596 & 1.41847 \\
\hline
\end{tabular}

Table 7: The result for the best fit of the profile of the lump with the gaussian curve described in eq.(4.9).

\section{Conclusions and Open Questions}

In this paper we have developed and tested the level expansion method in string field theory beyond translationally invariant vacuum solutions. This enabled us to give a systematic method for calculating quantities related to tachyon lumps and to give an accurate description of D-branes as tachyonic lumps in bosonic string field theory. Given the accuracy of our calculations (about 1\% typically) we are confident that the profile of the lump that we have found is indeed very close to the exact one. As we have seen, as long as the radius is sufficiently big the lump has a definite radius independent profile. Indeed, when approximated by a gaussian, the lump representing a D-brane has $\sigma \simeq 1.55 \sqrt{\alpha^{\prime}}$. We also considered the profile of the tachyon lump for $R=\sqrt{1.1 \alpha^{\prime}}$, a radius sufficiently small that the large radius profile of the lump does not fit on the circle. We saw that the bottom part of the lump is essentially unchanged.

There are some questions related to the present work that we have not addressed. In particular we have not produced a lump solution in string field theory for $R=1$, where the tachyon harmonic $t_{1}$ becomes exactly marginal and the D0 and D1 branes have the same mass. Presumably, for small $\left(R^{2}-1\right)$ one must go fairly high in the level expansion to produce an accurate description. We have also not discussed the case $R<1$, where the D0 brane is unstable against decay into the D1 brane, or into the translationally invariant vacuum. We have also not tried to describe several D0 
branes, all located at the same position.

We have not discussed issues related to the size of the lump representing a Dbrane. While in the conformal field theory description a D-brane is an object with a well defined position, in string field theory it is a fat object, with thickness of the order of the string scale. Since string field theory is a gauge theory one may wonder if the size is an artifact of the chosen gauge. We do not at present know the answer to this question. The simplest way to get some insight into the nature of this extended solution would be to try to find out the energy density. This fails since the string field theory action is nonlocal, and hence there is no known expression for energy density in this theory. It would be interesting to examine some physical question that could help interpret the nature of this size [34]. According to the conjectures of refs. [1], 2], all physical quantities calculated in the background of the lump solution must agree with those calculated in the background of a lower dimensional D-brane.

The methods used in this paper should be able to deal with:

- Neveu-Schwarz string field theory, where tachyon kinks rather than lumps represent lower dimensional D-branes. One way to deal with the boundary conditions on a circle would be to place both a kink and an anti-kink at diametrically opposite points of the circle. Another, probably more efficient way would be to include a Wilson line along the circle in such a way that the tachyon boundary conditions are twisted [5].

- Higher codimension D-branes. In [12] it was observed that as the codimension is increased the naive use of the tachyon "bounce" gave increasingly worse approximations to the lump mass. We believe that our methods will enable calculations to any desired accuracy. The simplest situation would involve making two of the original brane dimensions into circles and including harmonics in both directions by simple extension of the methods of section 2.2 .

- Intersecting D-branes. The simplest setup would be to begin with a D2-brane on a torus and generate a pair of transverse D1 branes intersecting at one point.

We hope that our analysis will ultimately provide a more refined understanding of string field theory and its geometry. One application is already apparent; if we could get 
a formulation of string field theory around the translationally invariant vacuum where the original D-brane is no longer present, such formulation will have more unbroken symmetries than the current formulation.

It is interesting to note that the level expansion method used here incorporates into the calculational scheme an ultra-violet (UV) cutoff. Since $l=p^{2}+\cdots$, working at fixed $l$ implies a upper bound to the momentum (in the spatial directions). From this one is naturally led to propose a level expansion method for quantum string field theory. One approach could be to use the Euclidean version of the theory, and make periodic all directions including time 35], thus turning, at any fixed level $M$, the set of all relevant fields into a set of expansion coefficients $c_{n}$, with $l\left(\left|\phi_{n}\right\rangle\right) \leq M$. Since we are setting the whole system in a box, we also have a natural infra-red cutoff. The whole quantum path integral $\int \prod\left[d c_{n}\right] \exp \left(-S\left(c_{n}\right) / \hbar\right)$ could then be evaluated. Alternatively, one could make all dimensions except time periodic. In this case the result would be the quantum mechanics of the wave functions $c_{n}(t)$. It would be exciting if the level expansion gave a concrete calculational definition of quantum string field theory, a definition one could in practice feed to a computer in order to calculate observables to any desired degree of precision.

Acknowledgements: We would like to thank R. Gopakumar, S. Minwalla, L. Rastelli and W. Taylor for discussions. A.S. acknowledges the Physics department of Harvard University and the Center for Theoretical Physics at MIT for hospitality during part of this work. The work of N.M. and B.Z. was supported in part by DOE contract \#DE-FC02-94ER40818.

\section{References}

[1] A. Sen, "Descent relations among bosonic D-branes," Int. J. Mod. Phys. A14, 4061 (1999) hep-th/9902105.

\footnotetext{
${ }^{9}$ Here $S$ should be the truncation, to the given level of approximation, of the full quantum action satisfying the exact quantum Batalin-Vilkovisky master equation. It is not clear that the cubic open string field theory provides such solution 36. The open-closed string field theory introduced in ref. [37] does provide a well defined quantum action, but due to the non-polynomial nature of the action it is not clear how to carry out the level expansion in this theory.
} 
[2] A. Recknagel and V. Schomerus, "Boundary deformation theory and moduli spaces of D-branes," Nucl. Phys. B545, 233 (1999) hep-th/9811237;

C.G. Callan, I.R. Klebanov, A.W. Ludwig and J.M. Maldacena, "Exact solution of a boundary conformal field theory," Nucl. Phys. B422, 417 (1994) hepth/9402113;

J. Polchinski and L. Thorlacius, "Free fermion representation of a boundary conformal field theory," Phys. Rev. D50, 622 (1994) hep-th/9404008.

[3] A. Sen, "Stable non-BPS bound states of BPS D-branes," JHEP 9808, 010 (1998) hep-th/9805019.

[4] A. Sen, "Tachyon condensation on the brane antibrane system," JHEP 9808, 012 (1998) hep-th/9805170.

[5] A. Sen, "SO(32) spinors of type I and other solitons on brane-antibrane pair," JHEP 9809, 023 (1998) hep-th/9808141.

[6] E. Witten, "D-branes and K-theory," JHEP 12, 019 (1998) hep-th/9810188.

[7] A. Sen, "BPS D-branes on non-supersymmetric cycles," JHEP 12, 021 (1998) hep-th/9812031.

[8] P. Horava, "Type IIA D-branes, K-theory, and matrix theory," Adv. Theor. Math. Phys. 2, 1373 (1999) hep-th/9812135.

[9] A. Sen and B. Zwiebach, "Tachyon Condensation in String Field Theory," hepth/9912249.

[10] W. Taylor, "D-brane effective field theory from string field theory", hepth/0001201.

[11] N. Moeller and W. Taylor, "Level truncation and the tachyon in open bosonic string field theory", hep-th/0002237.

[12] J.A. Harvey and P. Kraus, "D-Branes as unstable lumps in bosonic open string field theory", hep-th/0002117. 
[13] R. de Mello Koch, A. Jevicki, M. Mihailescu and R. Tatar, "Lumps and p-branes in open string field theory", hep-th/0003031.

[14] N. Berkovits, "The Tachyon Potential in Open Neveu-Schwarz String Field Theory," hep-th/0001084.

[15] N. Berkovits, A. Sen and B. Zwiebach, "Tachyon condensation in superstring field theory", hep-th/0002211.

[16] P. De Smet and J. Raeymaekers, "Level four approximation to the tachyon potential in superstring field theory", hep-th/0003220.

[17] A. Iqbal and A. Naqvi, "Tachyon condensation on a non-BPS D-brane," hepth/0004015.

[18] J. A. Harvey, P. Horava and P. Kraus, "D-sphalerons and the topology of string configuration space," hep-th/0001143.

[19] N. Drukker, D. J. Gross and N. Itzhaki, "Sphalerons, merons and unstable branes in AdS," hep-th/0004131.

[20] E. Witten, "Noncommutative Geometry And String Field Theory," Nucl. Phys. B268, 253 (1986).

[21] V.A. Kostelecky and S. Samuel, "The Static Tachyon Potential in the Open Bosonic String Theory," Phys. Lett. B207 (1988) 169;

V. A. Kostelecky and R. Potting, "Expectation Values, Lorentz Invariance, and CPT in the Open Bosonic String," Phys. Lett. B381, 89 (1996) hep-th/9605088.

[22] N. Berkovits, "Super-Poincare Invariant Superstring Field Theory," Nucl. Phys. B450 (1995) 90, hep-th/9503099.

[23] N. Berkovits, "A New Approach to Superstring Field Theory," proceedings to the $32^{\text {nd }}$ International Symposium Ahrenshoop on the Theory of Elementary Particles, Fortschritte der Physik (Progress of Physics) 48 (2000) 31, hep-th/9912121.

[24] N. Berkovits and C.T. Echevarria, "Four-Point Amplitude from Open Superstring Field Theory," hep-th/9912120. 
[25] O. Bergman, private communication.

[26] A. Iqbal and A. Naqvi, private communication.

[27] J. A. Harvey, D. Kutasov and E. J. Martinec, "On the relevance of tachyons," hep-th/0003101.

[28] P. Fendley, H. Saleur and N. P. Warner, "Exact solution of a massless scalar field with a relevant boundary interaction," Nucl. Phys. B430, 577 (1994) hepth/9406125].

[29] A. Sen, "Universality of the Tachyon Potential," hep-th/9911116.

[30] P. Ginsparg, "Applied Conformal Field Theory," HUTP-88-A054 Lectures given at Les Houches Summer School in Theoretical Physics, Les Houches, France, Jun 28 - Aug 5, 1988.

[31] M. R. Gaberdiel and B. Zwiebach, "Tensor constructions of open string theories I: foundations", Nucl. Phys. B505, 569 (1997) hep-th/9705038.

[32] D. Gross and A. Jevicki, "Operator formulation of interacting string field theory (I), (II)," Nucl. Phys. B283 (1987) 1, B287 (1987) 225;

E. Cremmer, A. Schwimmer and C. Thorn, "The vertex function in Witten's formulation of string field theory," Phys. Lett. B179 (1986) 57;

S. Samuel, "The physical and ghost vertices in Witten's string field theory,", Phys. Lett. B181 (1986) 255.

[33] A. LeClair, M.E. Peskin and C.R. Preitschopf, "String Field Theory On The Conformal Plane. 1. Kinematical Principles," Nucl. Phys. B317, 411 (1989); "String Field Theory On The Conformal Plane. 2. Generalized Gluing," Nucl. Phys. B317, 464 (1989).

[34] M. R. Douglas, D. Kabat, P. Pouliot and S. H. Shenker, "D-branes and short distances in string theory," Nucl. Phys. B485, 85 (1997) hep-th/9608024].

[35] G. Moore, "Finite in all directions," hep-th/9305139. 
[36] C. B. Thorn, "String field theory", Phys. Rep. 174 (1989) 1;

A. R. Bogojevic, "BRST Invariance of the measure in string field theory", Phys. Lett. B198 (1987) 479;

M. Maeno, "Canonical quantization of Witten's string field theory using midpoint light cone time", Phys. Rev. D43 (1991) 4006.

[37] B. Zwiebach, "Oriented open-closed string theory revisited," Annals Phys. 267, 193 (1998) hep-th/9705241. 